TRANSACTIONS OF THE

AMERICAN MATHEMATICAL SOCIETY

Volume 365, Number 3, March 2013, Pages 1183-1217

S 0002-9947(2012)05572-3

Article electronically published on August 9, 2012

\title{
SUPERCONDUCTIVITY NEAR THE NORMAL STATE IN A HALF-PLANE UNDER THE ACTION OF A PERPENDICULAR ELECTRIC CURRENT AND AN INDUCED MAGNETIC FIELD
}

\author{
YANIV ALMOG, BERNARD HELFFER, AND XING-BIN PAN
}

\begin{abstract}
We consider the linearization of the time-dependent GinzburgLandau system near the normal state. We assume that a constant magnetic field and an electric current are applied through the sample, which captures half of the plane, inducing thereby a linearly varying magnetic field. In the limit of small normal conductivity we prove that if the electric current is lower than some critical value, the normal state loses its stability. For currents stronger than this critical value, the normal state is stable. To obtain this stability result we analyze both the spectrum and the pseudo-spectrum of the linearized operator. The critical current tends, in this small conductivity limit, to another critical current which had been obtained for a reduced model which neglects magnetic field effects.
\end{abstract}

\section{Contents}

1. Introduction 1183

2. Analytic dilation $\quad 1189$

3. The scheme of the proof of the main results 1191

4. Constructions of quasimodes 1194

5. Resolvent estimates 1197

5.1. Preliminary estimates 1197

5.2. Projection on $\left(\operatorname{span}\left\{\psi_{0}\right\}\right)^{\perp} \quad 1206$

6. Existence, upper and lower bounds $\quad 1211$

7. Decay of the semi-group 1212

Appendix A. Some results related to pseudo-spectra

Acknowledgements $\quad 1216$

\begin{tabular}{ll} 
References & 1216 \\
\hline
\end{tabular}

\section{INTRODUCTION}

Consider a superconductor placed at a temperature lower than the critical one. If an electric current is applied through the sample it will induce a magnetic field, and as is well understood from numerous experimental observations [22], a sufficiently strong current will force the superconductor to arrive at the normal state. If the current is then lowered, the normal state would lose stability and the sample would

Received by the editors July 19, 2010 and, in revised form, February 20, 2011.

2010 Mathematics Subject Classification. Primary 82D55, 35B25, 35B40, 35Q55.

Key words and phrases. Superconductivity, critical current, critical magnetic field.

(C)2012 American Mathematical Society 
become superconducting again. In addition to experimental observations, a similar pattern of behaviour has also been obtained theoretically by analyzing the stability of the normal state for the time-dependent Ginzburg-Landau system, but with the induced magnetic field neglected [14, 3].

In a recent contribution 4 we analyzed the stability of the normal state in the presence of an electric current which induces a magnetic field, but in the absence of a boundary. We offered an analysis of a two-dimensional setting, i.e., in $\mathbb{R}^{2}$, which is the simplest case where one can consider the effect of a magnetic field induced by a current, with boundary effects ignored. We found in [4 that the normal state is always stable, irrespective of current intensity. This result is in line with those obtained for a reduced model where the magnetic field has been neglected 14, 3.

In the present contribution we introduce the effect of the boundary of the sample. With magnetic field neglected, this effect has been analyzed by considering a onedimensional problem on the half-line $\mathbb{R}_{+}=\{x \in \mathbb{R}: x>0\}$ with a Dirichlet boundary condition at $x=0$, which stands for a normal/superconducting interface 14, 3. Due to this effect, the normal state loses its stability when the current is weaker than a certain critical value. It has been proved in 3 that the critical current for a large bounded three-dimensional domain is bounded from above by the one-dimensional value. Furthermore, for a current below the critical one, a short-time instability was proven [3] (the question of whether the normal state is unstable for such domains and currents is still open).

We expect the boundary effect on the stability of the normal state in the presence of an induced magnetic field to be similar to their effect in the absence of magnetic fields [14, 3]. To examine the boundary's impact, we consider here a problem in the half-plane $\mathbb{R}_{+}^{2}$. If our expectation is correct, then in a similar fashion to [14, 3, the normal state would lose its stability for currents lower than some critical value, which we obtain here in the limit of small normal conductivity.

Assuming that a magnetic field described by $\mathcal{H}^{e}$ is perpendicularly applied to the sample, the time-dependent Ginzburg-Landau system can be written as follows (see for instance [5, 6, 9, 10, 14, 21, 23]):

$$
\begin{cases}\partial_{t} \psi+i \kappa \Phi \psi=\nabla_{\kappa \mathbf{A}}^{2} \psi+\kappa^{2}\left(1-|\psi|^{2}\right) \psi & \text { in }(0, T) \times \mathbb{R}_{+}^{2} \\ \kappa^{2} \operatorname{curl}^{2} \mathbf{A}+\sigma\left(\partial_{t} \mathbf{A}+\nabla \Phi\right)=\kappa \operatorname{Im}\left(\bar{\psi} \nabla_{\kappa \mathbf{A}} \psi\right)+\kappa^{2} \operatorname{curl} \mathcal{H}^{e} & \text { in }(0, T) \times \mathbb{R}_{+}^{2} \\ \psi=0, \quad-\frac{\sigma}{\kappa^{2}} \frac{\partial \Phi}{\partial \nu}=J & \text { on } \partial \mathbb{R}_{+}^{2}\end{cases}
$$

where $\psi$ is the order parameter, $\mathbf{A}$ is the magnetic potential, $\Phi$ is the electric potential, the Ginzburg-Landau parameter of the superconductor is denoted by $\kappa$, the normal conductivity of the sample is denoted by $\sigma$, the magnitude of the dimensionless electric current is denoted by $J$, and the applied magnetic field is denoted by $\mathcal{H}^{e}$. In (1.1) we use the notation

$$
\nabla_{\kappa \mathbf{A}} \psi=\nabla \psi-i \kappa \mathbf{A} \psi, \quad \nabla_{\kappa \mathbf{A}}^{2} \psi=\Delta \psi-i[2 \mathbf{A} \cdot \nabla \psi+\psi \operatorname{div} \mathbf{A}]-|\mathbf{A}|^{2} \psi,
$$

where $i=\sqrt{-1}$. The half-plane $\mathbb{R}_{+}^{2}$ is defined as

$$
\mathbb{R}_{+}^{2}=\left\{(x, y) \in \mathbb{R}^{2}: y>0\right\} .
$$

The triplet $(\psi, \mathbf{A}, \Phi)$ should also satisfy an initial condition at $t=0$.

A solution $(\psi, \mathbf{A}, \Phi)$ to (1.1) is called a normal state solution if $\psi \equiv 0$. From (1.1) we see that if $(0, \mathbf{A}, \Phi)$ is a time-independent normal state solution, then $(\mathbf{A}, \Phi)$ 
satisfies the following equation

$$
\kappa^{2} \operatorname{curl}^{2} \mathbf{A}+\sigma \nabla \Phi=\kappa^{2} \operatorname{curl}^{e} \quad \text { in } \mathbb{R}_{+}^{2} .
$$

By taking the divergence of (1.2) we obtain

$$
\begin{cases}\Delta \Phi=0 & \text { in } \mathbb{R}_{+}^{2}, \\ \frac{\partial \Phi}{\partial \nu}=-\frac{\kappa^{2} J}{\sigma} & \text { on } \partial \mathbb{R}_{+}^{2} .\end{cases}
$$

Since we expect solutions of (1.3) to represent the electric potential at the normal state near the boundary of a large bounded domain, we look for solutions with bounded gradient (or $\nabla \Phi \in L^{\infty}\left(\mathbb{R}_{+}^{2}\right)$ ). Assuming that the current is of constant magnitude $J$ along $\partial \mathbb{R}_{+}^{2}$ and that its direction is always perpendicular to it, we obtain that the unique solution to (1.3) which obeys these assumptions is given, up to an additive constant, by

$$
\Phi=\frac{\kappa^{2} J}{\sigma} y .
$$

Assuming further that the applied magnetic field is, like the current, of constant magnitude as well, we obtain

$$
\mathcal{H}^{e}=h \mathbf{i}_{z},
$$

throughout the entire sample. Here $\mathbf{i}_{x}, \mathbf{i}_{y}$, and $\mathbf{i}_{z}$ denote the canonical basis in $\mathbb{R}^{3}$. Hence, we consider an applied magnetic field which is perpendicular to the sample and parallel to its surface. Under these additional assumptions, equation (1.2) admits the following solution

$$
\mathbf{A}=\frac{1}{2 J}(J x+h)^{2} \mathbf{i}_{y} .
$$

For the above $\mathbf{A}$ and $\Phi,(0, \mathbf{A}, \Phi)$ is a normal state solution of (1.1). Note that the magnetic field

$$
\mathbf{H}=\operatorname{curl} \mathbf{A}=(J x+h) \mathbf{i}_{z},
$$

is the sum of the constant applied magnetic field $h \mathbf{i}_{z}$ and a linear term $J x \mathbf{i}_{z}$ induced by the electric current.

The linearization of (1.1) near the normal state solution $(0, \mathbf{A}, \Phi)$ obtained above yields a linear equation

$$
\begin{cases}\partial_{t} \psi+\frac{i \kappa^{3} J y}{\sigma} \psi=\Delta \psi-\frac{i \kappa}{J}(J x+h)^{2} \partial_{y} \psi-\left(\frac{\kappa}{2 J}\right)^{2}(J x+h)^{4} \psi+\kappa^{2} \psi & \text { in }(0, T) \times \mathbb{R}_{+}^{2}, \\ \psi=0 & \text { on } \partial \mathbb{R}_{+}^{2} .\end{cases}
$$

Applying the transformation

$$
(t, x, y) \rightarrow\left(t, x-\frac{h}{J}, y\right)
$$

we obtain

$$
\begin{cases}\partial_{t} \psi+\frac{i \kappa^{3} J y}{\sigma} \psi=\Delta \psi-i \kappa J x^{2} \partial_{y} \psi-\left(\left(\frac{\kappa J}{2}\right)^{2} x^{4}-\kappa^{2}\right) \psi & \text { in }(0, T) \times \mathbb{R}_{+}^{2}, \\ \psi=0 & \text { on } \partial \mathbb{R}_{+}^{2} .\end{cases}
$$

In the present contribution we analyze the asymptotic behaviour of the solutions of (1.7) for large $t$. We assume $J>0$ in the sequel. Otherwise we may either consider the complex conjugate of (1.7) or apply the transformation $y \rightarrow-y$. Hence, we can rescale $t, x$, and $y$ by applying

$$
t \rightarrow(\kappa J)^{2 / 3} t, \quad(x, y) \rightarrow(\kappa J)^{1 / 3}(x, y),
$$


yielding

$$
\begin{cases}\partial_{t} u=-\left(\mathcal{A}_{0, c}-\lambda\right) u & \text { in }(0, T) \times \mathbb{R}_{+}^{2}, \\ u=0 & \text { on } \partial \mathbb{R}_{+}^{2},\end{cases}
$$

where $\mathcal{A}_{0, c}$ is the differential operator defined by

$$
\mathcal{A}_{0, c}=D_{x}^{2}+\left(D_{y}-\frac{1}{2} x^{2}\right)^{2}+i c y
$$

with

$$
D_{x}=-i \partial_{x}, \quad D_{y}=-i \partial_{y}, \quad c \in \mathbb{R}_{+}
$$

and

$$
c=\frac{\kappa^{2}}{\sigma}, \quad \lambda=\lambda_{0} \equiv \frac{\kappa^{4 / 3}}{J^{2 / 3}}, \quad u(t, x, y)=\psi\left((\kappa J)^{-2 / 3} t,(\kappa J)^{-1 / 3} x,(\kappa J)^{-1 / 3} y\right) .
$$

As the operator $\mathcal{A}_{0, c}$ is defined on smooth functions only, it is necessary to extend its domain to also include weakly differentiable functions. We use the Lax-Milgram theorem to this end. Consider the sesquilinear form defined on $\tilde{V} \times \tilde{V}$ by

$$
(u, v) \longmapsto \tilde{a}(u, v)=\left\langle D_{x} u, D_{x} v\right\rangle+\left\langle\left(D_{y}-\frac{x^{2}}{2}\right) u,\left(D_{y}-\frac{x^{2}}{2}\right) v\right\rangle+i c \int_{0}^{+\infty} y u \bar{v} d x d y
$$

where

$$
\tilde{V}=H_{0}^{1, \operatorname{mag}}\left(\mathbb{R}_{+}^{2}, \mathbb{C}\right) \cap L^{2}\left(\mathbb{R}_{+}^{2}, \mathbb{C} ; y d x d y\right),
$$

and $H_{0}^{1, m a g}\left(\mathbb{R}_{+}^{2}, \mathbb{C}\right)$ is the closure of $C_{c}^{\infty}\left(\mathbb{R}_{+}^{2}, \mathbb{C}\right)$ under the norm

$$
u \longmapsto \sqrt{\|u\|^{2}+\left\|D_{x} u\right\|^{2}+\left\|\left(D_{y}-\frac{x^{2}}{2}\right) u\right\|^{2}} .
$$

Here and thereafter we use $L^{2}\left(\mathbb{R}_{+}^{2}\right)$ to denote the usual $L^{2}$ space of real-valued functions, $L^{2}\left(\mathbb{R}_{+}^{2}, \mathbb{C}\right)$ to denote the $L^{2}$ space of the complex-valued functions, and use $L^{2}\left(\mathbb{R}_{+}^{2} ; y d x d y\right)$ and $L^{2}\left(\mathbb{R}_{+}^{2}, \mathbb{C} ; y d x d y\right)$ to denote the related spaces with measure $y d x d y$. We use $\|\cdot\|$ and $\langle\cdot, \cdot\rangle$ to denote the $L^{2}$ norm and $L^{2}$ inner product on $\mathbb{R}_{+}^{2}$ :

$$
\|u\|=\|u\|_{L^{2}\left(\mathbb{R}_{+}^{2}\right)}=\left(\int_{\mathbb{R}_{+}^{2}}|u|^{2} d x\right)^{1 / 2}, \quad\langle u, v\rangle=\int_{\mathbb{R}_{+}^{2}} u \bar{v} d x .
$$

Denote further by $\tilde{q}$ the associated form

$$
\tilde{V} \ni u \longmapsto \tilde{q}(u)=\tilde{a}(u, u) .
$$

Observing that, when $c \neq 0$, there exists $\alpha>0$ such that

$$
\tilde{q}(u) \geq \alpha\|u\|_{\tilde{V}}^{2}, \quad \text { for all } u \in \tilde{V},
$$

we use the Lax-Milgram theorem to extend $\mathcal{A}_{0, c}$ into the unbounded operator $\mathcal{A}_{c}^{+}$ on $L^{2}\left(\mathbb{R}_{+}^{2}, \mathbb{C}\right)$ whose domain $D\left(\mathcal{A}_{c}^{+}\right)$is the set of the $u \in \tilde{V}$ such that the map

$$
C_{c}^{\infty}\left(\mathbb{R}_{+}^{2}, \mathbb{C}\right) \ni v \longmapsto \tilde{a}(u, v)
$$

extends as an anti-linear form on $L^{2}\left(\mathbb{R}_{+}^{2}, \mathbb{C}\right)$, which we denote by $\left\langle\mathcal{A}_{c}^{+} u, \cdot\right\rangle$. Hence we have

$$
D\left(\mathcal{A}_{c}^{+}\right)=\left\{u \in \tilde{V}: \mathcal{A}_{c}^{+} u \in L^{2}\left(\mathbb{R}_{+}^{2}, \mathbb{C}\right)\right\}
$$

and

$$
\tilde{a}(u, v)=\left\langle\mathcal{A}_{c}^{+} u, v\right\rangle, \quad \forall u \in D\left(\mathcal{A}_{c}^{+}\right), \quad \forall v \in \tilde{V} .
$$


Once the definition of the extended operator $\mathcal{A}_{c}^{+}$has been formulated, we may write

$$
\mathcal{A}_{c}^{+}=D_{x}^{2}+\left(D_{y}-\frac{1}{2} x^{2}\right)^{2}+i c y .
$$

Note that $\mathcal{A}_{c}^{+}$is not self-adjoint, but we have

$$
\left(\mathcal{A}_{c}^{+}\right)^{*}=\mathcal{A}_{-c}^{+} .
$$

In the present contribution we analyze the spectrum of $\mathcal{A}_{c}^{+}$, denoted by $\sigma\left(\mathcal{A}_{c}^{+}\right)$, and the semi-group associated with $\mathcal{A}_{c}^{+}$, which we denote by $\exp \left(-t \mathcal{A}_{c}^{+}\right)$.

By the methods of [4, we can easily prove the following:

Proposition 1.1. For any $c>0, \mathcal{A}_{c}^{+}$has a compact resolvent. Moreover, if $E_{0}(\beta)$ denotes the ground state energy of the anharmonic oscillator

and if

$$
-\frac{d^{2}}{d x^{2}}+\left(\frac{x^{2}}{2}+\beta\right)^{2}
$$

$$
E_{0}^{*}=\inf _{\beta \in \mathbb{R}} E_{0}(\beta),
$$

then

$$
\sigma\left(\mathcal{A}_{c}^{+}\right) \subset\left\{\lambda \in \mathbb{C}, \operatorname{Re} \lambda \geq E_{0}^{*}\right\} .
$$

As mentioned earlier, our interest is in the effect that the Dirichlet boundary condition in (1.9) has on the spectrum $\sigma\left(\mathcal{A}_{c}^{+}\right)$and on the semi-group $\exp \left(-t \mathcal{A}_{c}^{+}\right)$. Thus, it is interesting to compare them with the analogous entities for the wholeplane problem. Recall that in 4 we denoted by $\mathcal{A}$ the operator corresponding to the extension of $A_{0, c}$ from $C_{c}^{\infty}\left(\mathbb{R}^{2}, \mathbb{C}\right)$ to its closure under the associated graph norm.

Recall further, that for the whole plane problem, the spectrum of $\mathcal{A}$ is invariant under translations in a direction parallel to the imaginary axis. This property, together with the fact that the resolvent of $\mathcal{A}$ is compact, has the consequence that $\sigma(\mathcal{A})$ must be empty and that the decay of the semi-group $\exp (-t \mathcal{A})$ is faster than any exponential rate. On the other hand, for the half-plane problem discussed in the present paper, such an invariance principle does not hold for $\sigma\left(\mathcal{A}_{c}^{+}\right)$. Hence, we do not expect $\sigma\left(\mathcal{A}_{c}^{+}\right)$to be empty. Instead, we expect that eigenvalues of $\mathcal{A}_{c}^{+}$would exist, and that the system of corresponding eigenfunctions would be complete in $L^{2}\left(\mathbb{R}_{+}^{2}, \mathbb{C}\right)$. Here we provide a proof of the former statement in the asymptotic regime $c \rightarrow+\infty$, and leave the proof of the latter, as well as the discussion of the limit $c \rightarrow 0$, to future research.

The main results of this paper are the following:

Theorem 1.2. There exists $c_{0} \geq 0$ such that if $c \geq c_{0}$, then

$$
\sigma\left(\mathcal{A}_{c}^{+}\right) \neq \emptyset \text {. }
$$

Furthermore, there exists $\mu(c) \in \sigma\left(\mathcal{A}_{c}^{+}\right)$which behaves according to the following expansion as $c \rightarrow+\infty$ :

$$
\mu(c) \sim c^{2 / 3} \exp \left(i \frac{\pi}{3}\right) \alpha_{0}+\lambda_{1} \exp \left(-i \frac{\pi}{6}\right) c^{-1 / 3}+\mathcal{O}\left(c^{-5 / 6}\right),
$$

where $-\alpha_{0}$ is the rightmost zero point of the Airy's function [1, and $\lambda_{1}$ is given by (4.13). Finally, let

$$
\mu_{m}(c)=\inf _{z \in \sigma\left(\mathcal{A}_{c}^{+}\right)} \operatorname{Re} z
$$


Then, for all $c \geq c_{0}$ we have

$$
\mu_{m}(c) \sim \operatorname{Re} \mu(c)+\mathcal{O}\left(c^{-5 / 6}\right) .
$$

The lower bound of (1.18) is obtained in Proposition 3.5 whereas the upper bound is proved in Corollary 6.2 .

The next result is valid for all $c>0$.

Theorem 1.3. If $\sigma\left(\mathcal{A}_{c}^{+}\right) \neq \emptyset$, then

$$
\lim _{t \rightarrow+\infty}-\frac{\log \left\|\exp \left(-t \mathcal{A}_{c}^{+}\right)\right\|}{t}=\mu_{m}(c) .
$$

One can easily obtain from (1.16) and (1.18) the critical current $J_{c}$, for which the normal state loses its stability in the case when the electric current is perpendicular to the surface and the applied magnetic field is parallel to it. In fact for $0<\lambda<\mu_{m}$ the zero solution of (1.9) is stable, whereas for $\lambda>\mu_{m}$ it is unstable, as is manifested by Proposition 7.1 (see also [3] for the discussion of the case where the magnetic field is absent). From (1.16) and (1.18) we obtain that

$$
\frac{J_{c}}{\sigma}=c \mu_{m}^{-3 / 2}=\left[\frac{\alpha_{0}}{2}+\frac{\sqrt{3} \lambda_{1}}{2 c}+\mathcal{O}\left(c^{-3 / 2}\right)\right]^{-3 / 2} .
$$

Note that the critical current is independent of the applied magnetic field $\mathcal{H}^{e}=h \mathbf{i}_{z}$, whose sole effect is to translate the $x$ coordinate by $h / J$.

From (1.20) we see that for a large value of $c$

$$
\frac{J_{c}}{\sigma} \sim\left[\frac{\alpha_{0}}{2}\right]^{-3 / 2}\left[1-\frac{3 \sqrt{3} \lambda_{1}}{2 \alpha_{0} c}+\mathcal{O}\left(c^{-3 / 2}\right)\right] .
$$

When neglecting the induced magnetic field, the critical current can be evaluated for all $c>0$ (cf. [14, 3]). In this case we have

$$
\frac{J_{c}}{\sigma}=\left[\frac{\alpha_{0}}{2}\right]^{-3 / 2} \text {. }
$$

Consequently, one can persuasively argue that the simplified model, where the magnetic field is neglected [14, 3, 18, 17, 19, can be obtained from the linearized Ginzburg-Landau system in the limit $c \rightarrow+\infty$.

The above results significantly differ from those obtained previously for the problem in the entire plane [4. In the case of the half-plane the critical current is positive, meaning that the normal state becomes unstable for sufficiently small currents. In contrast, for the entire plane problem the normal state is stable for every non-zero current. This suggests that for a sample of finite size, if the current is lowered below some critical value, then instability would be initiated near the boundary. For the reduced model [14, where the magnetic field is neglected, it has been demonstrated numerically for a one-dimensional setting, that the emerging superconducting phase eventually captures the whole domain. The presence of a magnetic field may however have a stabilizing effect, and hence, one can expect a stable bifurcation of a thin surface layer of superconducting material from the normal state.

In this paper we focus our attention on the Dirichlet boundary condition $\psi=0$ on $\partial \mathbb{R}_{+}^{2}$ given in (1.1) as it represents a normal/superconducting interface. Other boundary conditions such as the Neumann condition $\frac{\partial \psi}{\partial \nu}=0$ on $\partial \mathbb{R}_{+}^{2}$, or the generalized Neumann condition $(\nabla-i \mathbf{A}) \psi \cdot \nu=0$, are also of great interest to mathematicians and physicists. 
As a final remark, note that the present contribution focuses on the special case where the electric current is perpendicular to the surface and the applied magnetic field is parallel to it. Though this case is encountered frequently in experiments where the potential is often constant on the surface, the more general case where the electric current and the applied magnetic field are arbitrarily directed is also of significant interest.

The rest of this work is arranged as follows: In the next section we transform $\mathcal{A}_{c}^{+}$into a new operator via analytic dilation and prove the relation between $\sigma\left(\mathcal{A}_{c}^{+}\right)$ and the spectrum of the transformed operator. In Section 3 we outline the scheme of the proof of Theorem 1.2. In Section 4, we use formal asymptotics to estimate the principal eigenvalue of $\mathcal{A}_{c}^{+}$and the corresponding eigenmode (the approximate eigenmode is usually called quasimode in the literature). To bound the error resulting from the approximation of the exact eigenmode by the quasimode, we prove in Section 5 some elliptic estimates. These estimates are used in Section 6 to complete the proof of Theorem 1.2. Finally, in Section 7, we prove Theorem 1.3

\section{Analytic Dilation}

In this section, we assume $c \neq 0$ and apply a technique inspired by the wisdom acquired during the study of the resonances of some Schrödinger operators by Aguilar and Combes [2] or Combes and Thomas [7] (see also Simon [20, pp. 498-500] for a survey and Davies [8, Lemma 5]).

For technical reasons it is easier to consider, instead of $\mathcal{A}_{c}^{+}$, the unitarily equivalent operator,

$$
\mathcal{P}=e^{-i x^{2} y / 2} \mathcal{A}_{c}^{+} e^{i x^{2} y / 2}=\left(D_{x}+x y\right)^{2}+D_{y}^{2}+i c y .
$$

Its associated sesquilinear form is given by

$$
a(u, v)=\left\langle\left(D_{x}+y x\right) u,\left(D_{x}+y x\right) v\right\rangle+\left\langle D_{y} u, D_{y} v\right\rangle+i c\left\langle y^{1 / 2} u, y^{1 / 2} v\right\rangle,
$$

whose domain is given by

$$
\begin{aligned}
V=\left\{u \in L^{2}\left(\mathbb{R}_{+}^{2}, \mathbb{C}\right):\left(D_{x}+y x\right) u \in L^{2}\left(\mathbb{R}_{+}^{2}, \mathbb{C}\right),\right. \\
\left.\quad D_{y} u \in L^{2}\left(\mathbb{R}_{+}^{2}, \mathbb{C}\right), y^{1 / 2} u \in L^{2}\left(\mathbb{R}_{+}^{2}, \mathbb{C}\right), u(\cdot, 0)=0\right\} .
\end{aligned}
$$

The condition $u(\cdot, 0)=0$ is to be understood in the sense of trace. Note that $V \subset H_{\text {loc }}^{1}\left(\overline{\mathbb{R}_{+}^{2}}, \mathbb{C}\right)$, hence every $u \in V$ has trace lying in $H_{\text {loc }}^{1 / 2}\left(\partial \mathbb{R}_{+}^{2}, \mathbb{C}\right)$. For later reference we note the obvious fact that $V$ is dense in $L^{2}\left(\mathbb{R}_{+}^{2}, \mathbb{C}\right)$.

Let $\theta \in \mathbb{C}$. Using a variant of the argument in 2, we introduce the dilation operator

$$
u \longmapsto(U(\theta) u)(x, y)=e^{-\theta / 2} u\left(e^{\theta} x, e^{-2 \theta} y\right)
$$

Then set

$$
\mathcal{P}_{\theta}:=U(\theta)^{-1} \mathcal{P} U(\theta)=e^{2 \theta}\left(D_{x}+y x\right)^{2}-e^{-4 \theta} \partial_{y}^{2}+i c e^{2 \theta} y,
$$

with the associated sesquilinear form $a_{\theta}$ given by

$$
\begin{aligned}
(u, v) \longmapsto a_{\theta}(u, v)= & e^{2 \theta}\left\langle\left(D_{x}+y x\right) u,\left(D_{x}+y x\right) v\right\rangle \\
& +e^{-4 \theta}\left\langle D_{y} u, D_{y} v\right\rangle+i c e^{2 \theta}\left\langle y^{1 / 2} u, y^{1 / 2} v\right\rangle .
\end{aligned}
$$


The domain of all sesquilinear forms $a_{\theta}$ is still given by $V \times V$ independently of $\theta$. We denote by $q_{\theta}$ the corresponding quadratic form,

$$
q_{\theta}(u)=a_{\theta}(u, u) .
$$

Lemma 2.1. If $\theta$ varies in $\mathbb{R}$, then the spectrum of $\mathcal{P}_{\theta}$ is independent of $\theta$.

Proof. Since $U(\theta)$ is unitary for all $\theta \in \mathbb{R}$, it follows from (2.3) that $\mathcal{P}_{\theta}$ is unitarily equivalent to $\mathcal{P}$ for all $\theta \in \mathbb{R}$. It follows that the spectrum of $\mathcal{P}_{\theta}$ is independent of $\theta$ if we restrict $\theta$ to the real line.

However, as we need to consider complex values of $\theta$ in the sequel, we have to first establish holomorphic dependence on $\theta \in \mathbb{C}$, in a sense to be made more precise (cf. [15]), of $\sigma\left(\mathcal{P}_{\theta}\right)$. To this end we first prove the following lemma.

Lemma 2.2. Let

$$
D_{0}=\left\{z \in \mathbb{C}:-\frac{\pi}{8}<\operatorname{Im} z<\frac{\pi}{24}\right\}
$$

Then, the forms

$$
\tilde{q}_{\theta}:=e^{-2 \theta-i \pi / 4} q_{\theta}, \quad \theta \in D_{0},
$$

constitute a holomorphic family of type (a).

Proof. For the precise definition of a type (a) holomorphic family of forms, the reader is referred to [15, Section VII.4.2]. To show that the forms (2.5) are of type (a), we have to show that, for all $\theta \in D_{0}$, the form $\tilde{q}_{\theta}$ given in (2.5) is sectorial and closed (see [15. pp. 310, 313]).

To show $\tilde{q}_{\theta}$ is sectorial, we need to estimate the size of the numerical range of the form $q_{\theta}$, namely the set

$$
\left\{z \in \mathbb{C}: \exists u \in V \text { with }\|u\|=1 \text { and } z=q_{\theta}(u)\right\} .
$$

For every $u \in V$ we have

$$
q_{\theta}(u)=e^{2 \theta}\left(\left\|\left(D_{x}+y x\right) u\right\|^{2}+i c\left\|y^{1 / 2} u\right\|^{2}\right)+e^{-4 \theta}\left\|\partial_{y} u\right\|^{2} .
$$

Consequently, we obtain that

$$
\min \{-4 \operatorname{Im} \theta, 2 \operatorname{Im} \theta\}=\alpha_{m} \leq \arg q_{\theta}(u) \leq \alpha_{M}=\max \left\{-4 \operatorname{Im} \theta, 2 \operatorname{Im} \theta+\frac{\pi}{2}\right\},
$$

and hence

$$
\left|\arg e^{-2 \theta-i \pi / 4} q_{\theta}(u)\right| \leq \max \left\{\frac{\pi}{4},\left|6 \operatorname{Im} \theta+\frac{\pi}{4}\right|\right\} .
$$

Since

$$
\left|6 \operatorname{Im} \theta+\frac{\pi}{4}\right|<\frac{\pi}{2}, \quad \forall \theta \in D_{0},
$$

we have

$$
\left|\arg \tilde{q}_{\theta}(u)\right|=\left|\arg e^{-2 \theta-i \pi / 4} q_{\theta}(u)\right|<\frac{\pi}{2}, \quad \forall \theta \in D_{0},
$$

from which sectoriality easily follows.

Finally, we need to verify that the form $\tilde{q}_{\theta}$ is closed. This is an immediate consequence of (2.7), the completeness of the space $H_{0}^{1, \mathrm{mag}}\left(\mathbb{R}_{+}^{2}, \mathbb{C}\right)$, and the definition of closeness for sesquilinear forms (cf. [15, Section VI.1.3]) . 
Remark 2.3. One can show that $\mathcal{P}_{\theta}$ is a type $(B)$ holomorphic family of operators (see [15, p. 395] for the definition) in the larger region

$$
-\frac{\pi}{6}<\operatorname{Im} \theta<\frac{\pi}{12} \text {. }
$$

For the purpose of this work, it suffices to prove this property for every $\theta \in D_{0}$, a fact which follows immediately from Lemma 2.2 and [15, Theorem VII.4.2].

Using the same technique as in the proof of [4, Proposition 2.4], the resolvent of $\mathcal{P}_{\theta}$ can be shown to be compact for every $\theta \in D_{0}$. Hence, we may use 15, Theorem VII.1.9] together with the fact, proved in Lemma 2.1, that $\sigma\left(\mathcal{P}_{\theta}\right)$ is independent of $\theta$ for $\theta \in \mathbb{R}$, to obtain the following:

Proposition 2.4. The spectrum of $\mathcal{P}_{\theta}$ is independent of $\theta$ in $D_{0}$.

As a corollary we get, by taking $\theta=-i \frac{\pi}{12}$, the following.

Corollary 2.5. The spectrum of $\mathcal{A}_{c}^{+}$satisfies

$$
\sigma\left(\mathcal{A}_{c}^{+}\right) \subset\left\{\lambda \in \mathbb{C}: \operatorname{Re} \lambda \geq \frac{1}{2}|c|^{2 / 3} \alpha_{0}\right\},
$$

where $\alpha_{0}$ is the ground-state energy of the Dirichlet realization of the Airy operator

$$
\mathcal{L}=D_{y}^{2}+y
$$

on $\mathbb{R}_{+}$.

We recall that $-\alpha_{0}$ is the rightmost zero point of the standard Airy function $A_{i}$, and

$$
\psi_{0}(y)=A_{i}\left(y-\alpha_{0}\right)
$$

is the eigenfunction of the operator $\mathcal{L}$ on $\mathbb{R}_{+}$with Dirichlet boundary condition at $y=0$, associated with the eigenvalue $\alpha_{0}$. More generally, if $-\alpha_{k} \in \mathbb{R}_{-}(k \in \mathbb{N})$ denotes the $(k+1)$-th zero of Airy's function, then

$$
\psi_{k}(y)=A_{i}\left(y-\alpha_{k}\right)
$$

is the eigenfunction of $\mathcal{L}$ on $\mathbb{R}_{+}$with Dirichlet boundary condition at $y=0$, associated with $\alpha_{k}$.

\section{The SChEME OF THE PROOF OF THE MAIN RESUlts}

In this section we outline the main steps of the proof of Theorem 1.2, Our goal is, thus, to obtain an estimate, in the large $c$ limit, for the real part of the leftmost eigenvalue of $\mathcal{A}_{c}^{+}$in $\mathbb{C}$. An immediate corollary would then be that $\sigma\left(\mathcal{A}_{c}^{+}\right) \neq \emptyset$.

Instead of dealing with $\sigma\left(\mathcal{A}_{c}^{+}\right)$, it is more convenient to analyze the spectrum of the operator $\mathcal{P}_{\theta}$ which is given by (2.3) and is obtained from $\mathcal{A}_{c}^{+}$using analytic dilation. For

$$
\theta=-i \frac{\pi}{12}
$$

we have

$$
\mathcal{P}_{-i \frac{\pi}{12}}=e^{i \pi / 3}\left(D_{y}^{2}+c y\right)+e^{-i \pi / 6}\left(D_{x}+x y\right)^{2} .
$$

Special emphasis should be given to the fact that $\mathcal{P}_{-i \frac{\pi}{12}}$ is not unitarily equivalent to $\mathcal{A}_{c}^{+}$. Hence, analytic dilation facilitates the analysis of the spectrum of $\mathcal{A}_{c}^{+}$, but the decay of the associated semi-group has to be obtained using a different 
approach. Since we consider large values of $c$, it is natural to introduce the small parameter

$$
\epsilon=\frac{1}{c}
$$

We define another operator via the (real) dilation

$$
\mathcal{B}_{\epsilon}:=\epsilon^{2 / 3} e^{i \pi / 6} U\left(-\ln \frac{\epsilon}{6}\right)^{-1} \mathcal{P}_{-i \frac{\pi}{12}} U\left(-\ln \frac{\epsilon}{6}\right),
$$

where $U$ is defined in (2.2). Explicitly we have

$$
\mathcal{B}_{\epsilon}=\epsilon\left(D_{x}+x y\right)^{2}+i\left(D_{y}^{2}+y\right) \text {. }
$$

The domain of $\mathcal{B}_{\epsilon}$ is

$$
D\left(\mathcal{B}_{\epsilon}\right)=\left\{u \in \tilde{V}: \mathcal{B}_{\epsilon} u \in L^{2}\left(\mathbb{R}_{+}^{2}, \mathbb{C}\right)\right\},
$$

where $\tilde{V}$ is given by (1.12).

From the foregoing discussion it follows that $\sigma\left(\mathcal{A}_{c}^{+}\right)$can easily be obtained from $\sigma\left(\mathcal{B}_{\epsilon}\right)$. In fact, any property we prove for the latter, including the next proposition, can be translated into a similar property of the former.

Proposition 3.1. There exists $\epsilon_{0}>0$ such that for $\epsilon \in\left(0, \epsilon_{0}\right)$ we have $\sigma\left(\mathcal{B}_{\epsilon}\right) \neq \emptyset$. Furthermore, there exists $\lambda \in \sigma\left(\mathcal{B}_{\epsilon}\right)$ such that

$$
\left|\lambda-i \alpha_{0}-\epsilon \lambda_{1}\right|<C \epsilon^{3 / 2} \text { for all } 0<\epsilon<\epsilon_{0},
$$

where $-\alpha_{0}$ is the rightmost zero point of the Airy's function [1, and $\lambda_{1}$ is given by (4.13) in Section 4.

The proof of the proposition is divided into four main steps.

Step 1. The first step entails the construction of quasimodes. Note that for a selfadjoint operator, a quasimode provides us with an upper bound for the bottom of the spectrum, via a variational principle, and hence the spectrum cannot be empty. For the non-self-adjoint operator $\mathcal{B}_{\epsilon}$, we construct the quasimodes in the next section, where we also obtain a formal asymptotic expansion, presented in Proposition 3.2, for the associated eigenvalue.

Denote by $\mathcal{S}\left(\overline{\mathbb{R}_{+}^{2}}\right)$ the space of the fast decay smooth functions defined on $\mathbb{R}_{+}^{2}$ (cf. [12] for the precise definition) and by $\mathcal{S}\left(\overline{\mathbb{R}_{+}^{2}}, \mathbb{C}\right)$ the corresponding space of complex-valued functions.

Proposition 3.2. There exist $u_{j}(j=0,1)$ in $\mathcal{S}\left(\overline{\mathbb{R}_{+}^{2}}, \mathbb{C}\right) \cap D\left(\mathcal{B}_{\epsilon}\right)$ with $\left\|u_{0}\right\|=1$ and $\lambda_{1} \in \mathbb{R}$, such that for

$$
\begin{cases}u_{\epsilon}^{\mathrm{app}}(x, y) & =u_{0}(x, y)+\epsilon u_{1}(x, y) \\ \gamma_{\epsilon} & =i \alpha_{0}+\epsilon \lambda_{1}\end{cases}
$$

we have

$$
\left(\mathcal{B}_{\epsilon}-\gamma_{\epsilon}\right) u_{\epsilon}^{\text {app }}=\epsilon^{2} f_{\epsilon}
$$

with $f_{\epsilon}$ being uniformly bounded in $\left(0, \epsilon_{0}\right)$ for any norm on $\mathcal{S}\left(\overline{\mathbb{R}_{+}^{2}}, \mathbb{C}\right)$. 
Proposition 3.2 is proved in Section 4

Remark 3.3. To describe the topology in $\mathcal{S}\left(\overline{\mathbb{R}_{+}^{2}}, \mathbb{C}\right)$, it is sufficient to use the family of norms

$$
f \longmapsto p_{k}(f)=\sum_{p+q+r+s \leq k}\left\|x^{p} y^{q} \partial_{x}^{r} \partial_{y}^{s} f\right\|,
$$

for $k \in \mathbb{N}$. We use this definition in the sequel whenever a norm appears in our calculations, except for the rare cases where the exact form of the norm should be introduced.

Step 2. We next show the existence of $\rho>0$ such that the circle

$$
\mathcal{C}_{\epsilon, \rho}=\left\{\lambda \in \mathbb{C}:\left|\lambda-\gamma_{\epsilon}\right|=\rho \epsilon^{3 / 2}\right\}
$$

does not intersect with the spectrum of $\mathcal{B}_{\epsilon}$ for sufficiently small $\epsilon$. Once this disjointedness is established, we can define the associated projector,

$$
\Pi_{\epsilon}=\frac{i}{2 \pi} \oint_{\mathcal{C}_{\epsilon, \rho}}\left(\mathcal{B}_{\epsilon}-\lambda\right)^{-1} d \lambda .
$$

Step 3. Then we show that the projector $\Pi_{\epsilon}$ does not identically vanish. To this end, it suffices to show that

$$
\Pi_{\epsilon} u_{\epsilon}^{\text {app }} \neq 0
$$

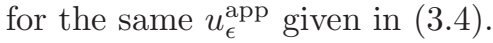

To prove (3.9) we represent 3.5 in the form

$$
u_{\epsilon}^{\mathrm{app}}=\frac{\epsilon^{2}}{\lambda-\gamma_{\epsilon}} f_{\epsilon}-\frac{1}{\lambda-\gamma_{\epsilon}}\left(\mathcal{B}_{\epsilon}-\lambda\right) u_{\epsilon}^{\mathrm{app}},
$$

which leads to

$$
i\left(\mathcal{B}_{\epsilon}-\lambda\right)^{-1} u_{\epsilon}^{\text {app }}=\frac{i \epsilon^{2}}{\lambda-\gamma_{\epsilon}}\left(\mathcal{B}_{\epsilon}-\lambda\right)^{-1} f_{\epsilon}-\frac{i}{\lambda-\gamma_{\epsilon}} u_{\epsilon}^{\text {app }} .
$$

Integrating the above identity with respect to $\lambda$ around $\mathcal{C}_{\epsilon, \rho}$ in the positive direction we obtain

$$
\Pi_{\epsilon} u_{\epsilon}^{\mathrm{app}}=u_{\epsilon}^{\mathrm{app}}+\frac{i \epsilon^{2}}{2 \pi} \oint_{\mathcal{C}_{\epsilon, \rho}} \frac{1}{\lambda-\gamma_{\epsilon}}\left(\mathcal{B}_{\epsilon}-\lambda\right)^{-1} f_{\epsilon} d \lambda .
$$

It then remains to show that the right-hand side of (3.10) does not identically vanish.

Step 4. Using (3.10) we see that to prove (3.9), it is sufficient to uniformly control the $L^{2}$ norm of $\left(\mathcal{B}_{\epsilon}-\lambda\right)^{-1} f_{\epsilon}$ for all $\lambda$ on the circle $\mathcal{C}_{\epsilon, \rho}$. In fact we prove the following.

Proposition 3.4. There exist positive constants $\rho, C$ and $\epsilon_{0}$ and $k_{0} \in \mathbb{N}$ such that, for all $\epsilon \in\left(0, \epsilon_{0}\right], \lambda \in \mathcal{C}_{\epsilon, \rho}$, and $f \in \mathcal{S}\left(\overline{\mathbb{R}_{+}^{2}}, \mathbb{C}\right)$, we have

$$
\left\|\left(\mathcal{B}_{\epsilon}-\lambda\right)^{-1} f\right\| \leq C \epsilon^{-3 / 2} p_{k_{0}}(f),
$$

where $p_{k_{0}}$ is the norm defined in (3.6). 
Proposition 3.4 will be proved at the end of Section 4 .

We can then conclude from (3.10) and (3.11) the existence of positive constants $C$ and $\epsilon_{0}$, such that, for any $\epsilon \in\left(0, \epsilon_{0}\right]$,

$$
\left\|\Pi_{\epsilon} u_{\epsilon}^{\mathrm{app}}\right\| \geq 1-C \epsilon^{1 / 2} .
$$

Then for small $\epsilon$ one can conclude (3.9) from (3.12).

To complete steps 2 and 4 , we analyze the equation,

$$
\left(\mathcal{B}_{\epsilon}-\lambda\right) w=f
$$

with $f \in \mathcal{S}\left(\overline{\mathbb{R}_{+}^{2}}, \mathbb{C}\right)$ and $w \in D\left(\mathcal{B}_{\epsilon}\right)$. We perform this task in Section 5 , where we derive various estimates of the solution $w$ of (3.13). In addition, we prove there the following result:

Proposition 3.5. Let

$$
D_{b, \epsilon}=\left\{\lambda \in \mathbb{C}: 0<\operatorname{Re} \lambda \leq \epsilon \lambda_{1}-b \epsilon^{3 / 2} \text { and } 0<\operatorname{Im} \lambda-\alpha_{0} \leq 3 \lambda_{1} \epsilon\right\} .
$$

Then, there exist positive $b_{0}$ and $\epsilon_{0}$ such that for all $\epsilon \in\left(0, \epsilon_{0}\right)$ and $b>b_{0}$ we have

$$
D_{b, \epsilon} \cap \sigma\left(\mathcal{B}_{\epsilon}\right)=\emptyset \text {. }
$$

Note that since

$$
\operatorname{Im}\left\langle\mathcal{B}_{\epsilon} u, u\right\rangle=\langle\mathcal{L} u, u\rangle \geq \alpha_{0}\|u\|^{2} \text { and } \operatorname{Re}\left\langle\mathcal{B}_{\epsilon} u, u\right\rangle \geq 0,
$$

it follows that

$$
\sigma\left(\mathcal{B}_{\epsilon}\right) \subset S_{b, \epsilon}
$$

where

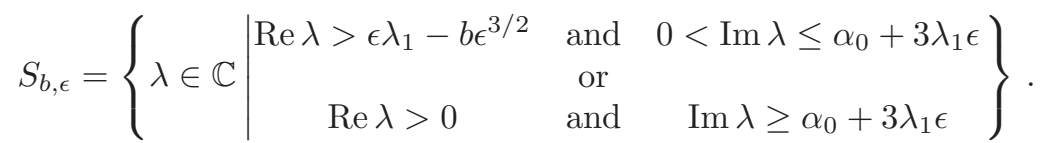

The lower bound in (1.18) now immediately follows from (3.15) and (3.16).

Proposition 3.5 is proved in Section 6

\section{Constructions of quasimodes}

The main result in this section is Proposition 4.1 which implies Proposition 3.2 in Section 3

Proposition 4.1. Let $\mathcal{B}_{\epsilon}$ be the operator defined in (3.2). There exists a sequence

$$
\left\{u_{j}(x, y)\right\}_{j=0}^{+\infty} \subset \mathcal{S}\left(\overline{\mathbb{R}_{+}^{2}}, \mathbb{C}\right) \cap D\left(\mathcal{B}_{\epsilon}\right)
$$

with $\left\|u_{0}\right\|_{L^{2}}=1$ and a sequence

$$
\left\{\lambda_{j}\right\}_{j=0}^{+\infty} \subset \mathbb{C}
$$

with $i^{j+1} \lambda_{j}$ being real for all $j \geq 0$ and

$$
\lambda_{0}=i \alpha_{0}
$$

where $\alpha_{0}$ is the lowest eigenvalue of the Airy operator $\mathcal{L}$ defined in (2.10) in $\mathbb{R}_{+}$ with homogeneous Dirichlet condition at $y=0$, such that

$$
\mathcal{B}_{\epsilon}\left(\sum_{j=0}^{+\infty} \epsilon^{j} u_{j}(x, y)\right) \sim\left(\sum_{j=0}^{+\infty} \epsilon^{j} \lambda_{j}\right)\left(\sum_{j=0}^{+\infty} \epsilon^{j} u_{j}(x, y)\right)
$$

in the sense of formal expansions in $\epsilon$. 
Remark 4.2. We note that if $\epsilon$ had been purely imaginary, then by the spectral theorem (4.3) would have implied the existence of an eigenvalue $\lambda(\epsilon)$ admitting the complete asymptotic expansion

$$
\lambda(\epsilon) \sim \sum_{j=0}^{+\infty} \epsilon^{j} \lambda_{j} .
$$

Since our interest is in the non-self-adjoint case where $\epsilon$ is real, a more complicated approach has to be adopted in order to prove the existence of an eigenvalue of $\mathcal{B}_{\epsilon}$.

Proof. It is sufficient to show how one can obtain $u_{j}$ and $\lambda_{j}$ for $j \leq 2$. Higher order terms can similarly be obtained. We first derive the $\mathcal{O}\left(\epsilon^{j}\right)$ balance from (4.3) for every $j \geq 0$. Suppose that the sequence in (4.1) satisfies (4.3) in the sense of formal expansions in $\epsilon$. Balancing the coefficients of $\epsilon^{j}$ on each side of (4.3), we obtain

$$
\begin{aligned}
& i\left(D_{y}^{2}+y\right) u_{0}=\lambda_{0} u_{0}, \\
& i\left(D_{y}^{2}+y\right) u_{j}+\left(D_{x}+x y\right)^{2} u_{j-1}=\sum_{\ell+m=j} \lambda_{\ell} u_{m}, \quad j \geq 1 .
\end{aligned}
$$

Step 1 . The $\mathcal{O}\left(\epsilon^{0}\right)$ balance gives

$$
\left(i\left(D_{y}^{2}+y\right)-\lambda_{0}\right) u_{0}(x, y)=0 .
$$

It follows immediately that $\lambda_{0}=i \alpha_{0}$, in accordance with (4.2). Furthermore, it follows that $u_{0}$ must be in the form

$$
u_{0}(x, y)=\phi_{0}(x) \psi_{0}(y),
$$

where $\psi_{0}(y)$ is the $L^{2}$-normalized (real-valued) first eigenfunction of the Airy operator $\mathcal{L}=D_{y}^{2}+y$, defined in (2.10), in $\mathbb{R}_{+}$with Dirichlet boundary at $y=0$, associated with the lowest eigenvalue $\alpha_{0}$. It is well known that $\psi_{0}$ is in $\mathcal{S}\left(\overline{\mathbb{R}_{+}}\right)$(see 1). We leave $\phi_{0}$ undetermined as it cannot be obtained from (4.5).

Step 2. The $\mathcal{O}(\epsilon)$ balance reads

$$
i\left(\left(D_{y}^{2}+y\right)-\alpha_{0}\right) u_{1}(x, y)+\left(D_{x}+x y\right)^{2} u_{0}(x, y)=\lambda_{1} u_{0}(x, y) .
$$

A necessary condition for the solvability of (4.7) is obtained by taking the scalar product (in the $y$-variable) of both sides of (4.7) by $\psi_{0}$. Since $u_{1} \in \mathcal{S}\left(\overline{\mathbb{R}_{+}^{2}}, \mathbb{C}\right) \cap D\left(\mathcal{B}_{\epsilon}\right)$ we have that $u_{1}(x, 0)=0$. Integration by parts then yields

$$
\int_{0}^{+\infty}\left[\left(D_{y}^{2}+y-\alpha_{0}\right) u_{1}\right] \bar{\psi}_{0} d y=0
$$

Since $\left\|\psi_{0}\right\|_{L^{2}\left(\mathbb{R}_{+}\right)}=1$, we have that

$$
\int_{0}^{+\infty}\left[\left(D_{x}+x y\right)^{2} \phi_{0}(x)\right] \psi_{0}(y)^{2} d y=\mathcal{K}_{0} \phi_{0}(x),
$$

where $\mathcal{K}_{0}$ is the differential operator

$$
\mathcal{K}_{0}\left(x, D_{x}\right)=\left(D_{x}+\alpha x\right)^{2}+\left(\beta^{2}-\alpha^{2}\right) x^{2}, \quad x \in \mathbb{R},
$$

in which

$$
\alpha=\int_{0}^{+\infty} y \psi_{0}(y)^{2} d y, \quad \beta^{2}=\int_{0}^{+\infty} y^{2} \psi_{0}(y)^{2} d y .
$$


Combining (4.7), (4.8), and (4.9) gives

$$
\mathcal{K}_{0} \phi_{0}(x)=\lambda_{1} \phi_{0}(x) \text {. }
$$

Since $y \psi_{0}(y)$ is not a multiple of $\psi_{0}$, it follows from Cauchy-Schwarz inequality that the following strict inequality holds:

$$
\alpha<\beta \text {. }
$$

Hence $\mathcal{K}_{0}$ is an harmonic oscillator, whose lowest eigenvalue is $\sqrt{\beta^{2}-\alpha^{2}}$. Consequently,

$$
\lambda_{1}=\sqrt{\beta^{2}-\alpha^{2}},
$$

and $\phi_{0}$ is the corresponding $L^{2}$-normalized eigenfunction. Explicitly, $\phi_{0}$ is a complex Gaussian,

$$
\phi_{0}(x)=a \exp \left(\frac{i \alpha}{2} x^{2}\right) \exp \left(-\frac{1}{2} \sqrt{\beta^{2}-\alpha^{2}} x^{2}\right),
$$

where the constant $a$ is chosen so that $\left\|\phi_{0}\right\|_{L^{2}(\mathbb{R})}=1$.

The solvability of (4.7) now easily follows: since $\mathcal{L}$ is self-adjoint, and all its eigenvalues are simple, it follows that

$$
\left(D_{x}+x y\right)^{2} u_{0}(x, y)-\lambda_{1} u_{0}(x, y) \perp \operatorname{Ker}\left\{\mathcal{L}-\alpha_{0}\right\}, \quad \forall x \in \mathbb{R} .
$$

As $\mathcal{L}-\alpha_{0}$ is a Fredholm operator with index zero, the solvability of (4.7) readily follows. Consequently, $u_{1}$ is expressible in the form

$$
u_{1}(x, y)=u_{1}^{0}(x, y)+\phi_{1}(x) \psi_{0}(y)
$$

where $u_{1}^{0}$ is the unique solution of (4.7) belonging to $\left(\operatorname{span}\left\{\psi_{0}\right\}\right)^{\perp}$ for all $x \in \mathbb{R}$, i.e.,

$$
\int_{0}^{+\infty} u_{1}^{0}(x, y) \psi_{0}(y) d y=0 .
$$

Step 3. Consider next the $\mathcal{O}\left(\epsilon^{2}\right)$ balance,

(4.17) $i\left(\left(D_{y}^{2}+y\right)-\alpha_{0}\right) u_{2}(x, y)+\left(D_{x}+x y\right)^{2} u_{1}(x, y)=\lambda_{1} u_{1}(x, y)+\lambda_{2} u_{0}(x, y)$.

A first necessary condition for solving (4.17) is obtained by taking the scalar product in the $y$-variable of both sides of (4.17) with $\psi_{0}$. Using (4.8) and (4.15), we obtain

$$
\int_{0}^{+\infty}\left[\left(D_{x}+x y\right)^{2} u_{1}(x, y)\right] \psi_{0}(y) d y=\mathcal{K}_{0} \phi_{1}(x)+\int_{0}^{+\infty}\left[\left(D_{x}+x y\right)^{2} u_{1}^{0}(x, y)\right] \psi_{0}(y) d y .
$$

Combining the above with (4.16) then yields

$$
\left(\mathcal{K}_{0}-\lambda_{1}\right) \phi_{1}=\lambda_{2} \phi_{0}-\left\langle\psi_{0},\left(\left(D_{x}+x y\right)^{2}-\lambda_{1}\right) u_{1}^{0}(x, y)\right\rangle_{L^{2}\left(\mathbb{R}_{+}\right)} .
$$

A second solvability condition is obtained by taking the $L^{2}$ scalar product of both sides of (4.18) with $\phi_{0}$ in the $x$-variable. Using (4.11), we arrive at

$$
\int_{\mathbb{R}}\left[\left(\mathcal{K}_{0}-\lambda_{1}\right) \phi_{1}\right] \phi_{0} d x=\int_{\mathbb{R}}\left[\left(\mathcal{K}_{0}-\lambda_{1}\right) \phi_{0}\right] \phi_{1} d x=0 .
$$

Hence,

$$
\begin{aligned}
\lambda_{2} & =\int_{\mathbb{R}}\left\langle\psi_{0},\left(\left(D_{x}+x y\right)^{2}-\lambda_{1}\right) u_{1}^{0}(x, y)\right\rangle_{L^{2}\left(\mathbb{R}_{+}\right)} \phi_{0} d x \\
& =\left\langle u_{0},\left(\left(D_{x}+x y\right)^{2}-\lambda_{1}\right) u_{1}^{0}(x, y)\right\rangle_{L^{2}\left(\mathbb{R}_{+}^{2}\right)} .
\end{aligned}
$$


With this choice of $\lambda_{2}$, (4.18) satisfies the necessary condition for solvability, which is also a sufficient condition. We can then choose $\phi_{1}$ to be the unique solution of (4.18) which is orthogonal to $\phi_{0}$.

Substituting $\phi_{1}$ into (4.15) we obtain $u_{1}$. Then (4.17) satisfies the necessary condition for solvability, which is also sufficient by the same argument applied in Step 2. Thus, the solution of (4.17) assumes the form

$$
u_{2}(x, y)=u_{2}^{0}(x, y)+\phi_{2}(x) \psi_{0}(y)
$$

where $u_{2}^{0}$ is uniquely determined by the orthogonality condition

$$
\int_{0}^{+\infty} u_{2}^{0}(x, y) \psi_{0}(y) d y=0 .
$$

It is easy to show that the $u_{j}$ 's are all in $\mathcal{S}\left(\overline{\mathbb{R}_{+}^{2}}, \mathbb{C}\right)$ in view of the fact that both $\mathcal{L}$ and $\mathcal{K}_{0}$ include an unbounded potential as $y$ and $|x|$, respectively, tend to infinity.

This completes the proof of Proposition 4.1.

Proof of Proposition 3.2. Proposition 3.2 readily follows from Proposition 4.1

\section{Resolvent estimates}

Our goal in this section is to prove Proposition 3.4 by estimating the resolvent of $\mathcal{B}_{\epsilon}$ for $\lambda \in D_{b, \epsilon}$, where $D_{b, \epsilon} \subset \mathbb{C}$ was given by (3.14).

5.1. Preliminary estimates. We begin by establishing the following approximation.

Lemma 5.1. Let $\phi$ be a real-valued $C^{\infty}$ function which together with its derivatives is bounded for $x \in \mathbb{R}$, and $w \in \mathcal{S}\left(\overline{\mathbb{R}_{+}^{2}}, \mathbb{C}\right) \cap D\left(\mathcal{B}_{\epsilon}\right)$. Then

$$
\int_{\mathbb{R}_{+}^{2}} \phi(x)^{2}\left(\mathcal{B}_{\epsilon} w\right) \bar{w} d x d y=\left\|\phi D_{y} w\right\|^{2}+\left\|y^{1 / 2} \phi w\right\|^{2}+\epsilon W,
$$

where $W \in L^{2}\left(\mathbb{R}_{+}^{2}, \mathbb{C}\right)$ satisfies

$$
\|W\| \leq\left\|\left(D_{x}+x y\right)(\phi w)\right\|^{2}+\left\|\phi^{\prime} w\right\|^{2} .
$$

Proof. Since $w$ vanishes on $\partial \mathbb{R}_{+}^{2}$, we can integrate by parts and the term involving the integral on the boundary $\partial \mathbb{R}_{+}^{2}$ vanishes. We therefore have

$$
\begin{aligned}
\int_{\mathbb{R}_{+}^{2}} \phi(x)^{2}\left(\mathcal{B}_{\epsilon} w\right) \bar{w} d x d y= & \epsilon \int_{\mathbb{R}_{+}^{2}}\left\{\left|\phi\left(D_{x}+x y\right) w\right|^{2}+2 i \phi \phi^{\prime} \bar{w}\left(D_{x}+x y\right) w\right\} d x d y \\
& +i \int_{\mathbb{R}_{+}^{2}}\left(\left|\phi D_{y} w\right|^{2}+y|\phi w|^{2}\right) d x d y
\end{aligned}
$$

Taking the imaginary part of $(\underline{5.3})$ we obtain

$$
\begin{aligned}
& \operatorname{Im} \int_{\mathbb{R}_{+}^{2}} \phi(x)^{2}\left(\mathcal{B}_{\epsilon} w\right) \bar{w} d x d y \\
= & \left\|\phi D_{y} w\right\|^{2}+\left\|y^{1 / 2} \phi w\right\|^{2}+2 \epsilon \operatorname{Re} \int_{\mathbb{R}_{+}^{2}} \phi \phi^{\prime}\left[\bar{w}\left(D_{x}+x y\right) w\right] d x d y .
\end{aligned}
$$


The integral in the last term in the right-hand side can be rewritten as

$$
\operatorname{Re} \int_{\mathbb{R}_{+}^{2}} \phi \phi^{\prime}\left[\bar{w}\left(D_{x}+x y\right) w\right] d x d y=\operatorname{Re} \int_{\mathbb{R}_{+}^{2}} \phi^{\prime}\left[\bar{w}\left(D_{x}+x y\right)(\phi w)\right] d x d y .
$$

Consequently, we may conclude that

$$
\left|\operatorname{Re} \int_{\mathbb{R}_{+}^{2}} \phi \phi^{\prime}\left[\bar{w}\left(D_{x}+x y\right) w\right] d x d y\right| \leq\left\|\left(D_{x}+x y\right)(\phi w)\right\|\left\|\phi^{\prime} w\right\| .
$$

For the real part of the integral on the left-hand side of (5.1) we have

$$
\begin{aligned}
& \operatorname{Re} \int(x)^{2}\left(\mathcal{B}_{\epsilon} w\right) \bar{w} d x d y \\
= & \epsilon \int_{\mathbb{R}_{+}^{2}}\left\{\left|\phi\left(D_{x}+x y\right) w\right|^{2}+i \phi \phi^{\prime}\left[\bar{w}\left(D_{x}+x y\right) w-w \overline{\left(D_{x}+x y\right) w}\right]\right\} d x d y \\
= & \epsilon\left\|\phi\left(D_{x}+x y\right) w-i \phi^{\prime} w\right\|^{2}-\epsilon\left\|\phi^{\prime} w\right\|^{2}=\epsilon\left\|\left(D_{x}+x y\right)(\phi w)\right\|^{2}-\epsilon\left\|\phi^{\prime} w\right\|^{2} .
\end{aligned}
$$

Combining (5.5) and (5.6) yields

$\left|\int_{\mathbb{R}_{+}^{2}} \phi(x)^{2}\left(\mathcal{B}_{\epsilon} w\right) \bar{w} d x d y-\left\|\phi D_{y} w\right\|^{2}+\left\|y^{1 / 2} \phi w\right\|^{2}\right| \leq 2 \epsilon\left(\left\|\left(D_{x}+x y\right)(\phi w)\right\|^{2}+\left\|\phi^{\prime} w\right\|^{2}\right)$,

which completes the proof of the lemma.

We continue by establishing the following estimates, which are valid for $\lambda$ values in a much greater range than those proved in the next subsection.

Lemma 5.2. Let $(f, w) \in S\left(\overline{\mathbb{R}_{+}^{2}}, \mathbb{C}\right) \times D\left(\mathcal{B}_{\epsilon}\right)$ satisfy (3.13). Given any $C>0$, there exist constants $\epsilon_{0}$ and $\widehat{C}$, and, for any half-integer $k \geq 1 / 2$, a constant $C_{k}$, such that, for any

$$
\lambda=i \alpha_{0}+\epsilon \mu_{1}
$$

with

$$
\left|\mu_{1}\right| \leq C, \quad \epsilon \in\left(0, \epsilon_{0}\right]
$$

we have

$$
|x|^{k} w \in L^{2}\left(\mathbb{R}_{+}^{2}, \mathbb{C}\right), \quad|y|^{k} w \in L^{2}\left(\mathbb{R}_{+}^{2}, \mathbb{C}\right), \quad x \partial_{x} w \in L^{2}\left(\mathbb{R}_{+}^{2}, \mathbb{C}\right),
$$

with

$$
\begin{gathered}
\left\||x|^{k} w\right\| \leq C_{k}\left[\|w\|+\epsilon^{-1}\left\|\left(|x|^{k-1 / 2}+1\right) f\right\|\right], \\
\left\|y^{k} w\right\| \leq C_{k}\left[\|w\|+\left\|\left(y^{k-1 / 2}+1\right) f\right\|\right], \\
\left\||x|^{k} \partial_{x} w\right\| \leq C_{k}\left[\|w\|+\epsilon^{-1}\left\|\left(|x|^{k+3 / 2}+y^{k+3 / 2}+1\right) f\right\|\right], \\
\left\|y^{k} \partial_{x} w\right\| \leq C_{k}\left[\|w\|+\epsilon^{-1}\left\|\left(|x|^{5 / 2}+y^{k+1}+1\right) f\right\|+\left\|y^{k-1 / 2} \partial_{x} f\right\|\right], \\
\left\|\partial_{x x} w\right\| \leq \widehat{C}\left[\|w\|+\epsilon^{-1}\left\|\left(|x|^{7 / 2}+y^{7 / 2}+1\right) f\right\|+\left\|y^{3 / 2} \partial_{x} f\right\|+\epsilon^{-1}\left\|\partial_{x} f\right\|\right] .
\end{gathered}
$$

Proof. Step 1. We first obtain an estimate of $\left\|\left(\nabla+i x y \hat{i_{y}}\right) w\right\|$. 
To this end, let $v \in \tilde{V}$ be compactly supported in the interior of $\mathbb{R}_{+}^{2}$, where $\tilde{V}$ was defined in (1.12). Multiplying (3.13) by $\bar{v}$ and integrating by parts yields $(5.10)$

$\int_{\mathbb{R}_{+}^{2}}\left\{\epsilon\left(D_{x}+x y\right) w \cdot \overline{\left(D_{x}+x y\right) v}+i D_{y} w \cdot \overline{D_{y} v}+i y w \bar{v}-\lambda w \bar{v}\right\} d x d y=\int_{\mathbb{R}_{+}^{2}} f \bar{v} d x d y$.

For $n \geq 1$, let $\eta_{n} \in C^{\infty}(\mathbb{R},[0,1])$ satisfy

$$
\eta_{n}(t)= \begin{cases}0 & \text { if }|t|<1 \\ 1 & \text { if } 2<|t|<4 n, \\ 0 & \text { if } 6 n<|t|,\end{cases}
$$

and

$$
\left|\eta_{n}^{\prime}\right| \leq \frac{C_{0}}{n}
$$

Let $m \geq 0$ and set $v(x, y)=|x|^{m} \eta_{n}^{2}(x) w(x, y)$ in (5.10). We compute

$$
\begin{aligned}
\left(D_{x}+x y\right) w \cdot \overline{\left(D_{x}+x y\right) v}= & \left|\left(D_{x}+x y\right)\left(|x|^{m / 2} \eta_{n} w\right)\right|^{2}-\left|w\left(|x|^{m / 2} \eta_{n}\right)^{\prime}\right|^{2} \\
& +2 i|x|^{m / 2} \eta_{n}\left(|x|^{m / 2} \eta_{n}\right)^{\prime} \operatorname{Re}\left[\bar{w}\left(D_{x}+x y\right) w\right] .
\end{aligned}
$$

We can thus write (5.10) in the form

$$
\begin{aligned}
& \epsilon \int_{\mathbb{R}_{+}^{2}}\left\{\left|\left(D_{x}+x y\right)\left(|x|^{m / 2} \eta_{n} w\right)\right|^{2}-\left|w\left(|x|^{m / 2} \eta_{n}\right)^{\prime}\right|^{2}\right\} d x d y \\
& +i \int_{\mathbb{R}_{+}^{2}}\left\{2 \epsilon|x|^{m / 2} \eta_{n}\left(|x|^{m / 2} \eta_{n}\right)^{\prime} \operatorname{Re}\left[\bar{w}\left(D_{x}+x y\right) w\right]\right. \\
& \left.\quad+|x|^{m} \eta_{n}^{2}\left|D_{y} w\right|^{2}+y|x|^{m} \eta_{n}^{2}|w|^{2}\right\} d x d y \\
& -\left(i \alpha_{0}+\epsilon \mu_{1}\right) \int_{\mathbb{R}_{+}^{2}}|x|^{m} \eta_{n}^{2}|w|^{2} d x d y=\int_{\mathbb{R}_{+}^{2}}|x|^{m} \eta_{n}^{2} f \bar{w} d x d y .
\end{aligned}
$$

Here we have used the assumption (5.8).

Taking the real part of (5.12) we get

$$
\begin{aligned}
& \epsilon \int_{\mathbb{R}_{+}^{2}}\left\{\left|\left(D_{x}+x y\right)\left(|x|^{m / 2} \eta_{n} w\right)\right|^{2}-\left|w\left(|x|^{m / 2} \eta_{n}\right)^{\prime}\right|^{2}-\operatorname{Re} \mu_{1}|x|^{m} \eta_{n}^{2}|w|^{2}\right\} d x d y \\
& =\operatorname{Re} \int_{\mathbb{R}_{+}^{2}}|x|^{m} \eta_{n}^{2} f \bar{w} d x d y .
\end{aligned}
$$

Hence we have

$$
\begin{aligned}
& \int_{\mathbb{R}_{+}^{2}}\left|\left(D_{x}+x y\right)\left(|x|^{m / 2} \eta_{n}(x) w\right)\right|^{2} d x d y \\
= & \operatorname{Re} \mu_{1}\left\||x|^{m / 2} \eta_{n} w\right\|^{2}+\left\|\mid\left(|x|^{m / 2} \eta_{n}\right)^{\prime} w\right\|^{2}+\epsilon^{-1} \operatorname{Re}\left\langle|x|^{m / 2} \eta_{n} w,|x|^{m / 2} \eta_{n} f\right\rangle .
\end{aligned}
$$

Taking the imaginary part of (5.12) yields

$$
\begin{aligned}
& \int_{\mathbb{R}_{+}^{2}}\left\{2 \epsilon|x|^{m / 2} \eta_{n}\left(|x|^{m / 2} \eta_{n}\right)^{\prime} \operatorname{Re}\left[\bar{w}\left(D_{x}+x y\right) w\right]\right. \\
& \left.+|x|^{m} \eta_{n}^{2}\left|D_{y} w\right|^{2}+y|x|^{m} \eta_{n}^{2}|w|^{2}\right\} d x d y \\
& -\left(\alpha_{0}+\epsilon \operatorname{Im} \mu_{1}\right) \int_{\mathbb{R}_{+}^{2}}|x|^{m} \eta_{n}^{2}|w|^{2} d x d y=\operatorname{Im} \int_{\mathbb{R}_{+}^{2}}|x|^{m} \eta_{n}^{2} f \bar{w} d x d y .
\end{aligned}
$$


Hence,

$$
\begin{aligned}
& \left\||x|^{m / 2} \eta_{n} D_{y} w\right\|^{2}+\left\|y^{1 / 2}|x|^{m / 2} \eta_{n} w\right\|^{2} \\
= & \left(\alpha_{0}+\epsilon \operatorname{Im} \mu_{1}\right)\left\||x|^{m / 2} \eta_{n} w\right\|^{2}+\operatorname{Im}\left\langle\eta_{n}|x|^{m / 2} w, \eta_{n}|x|^{m / 2} f\right\rangle \\
& -2 \epsilon \int_{\mathbb{R}_{+}^{2}}|x|^{m / 2} \eta_{n}\left(|x|^{m / 2} \eta_{n}\right)^{\prime} \operatorname{Re}\left[\bar{w}\left(D_{x}+x y\right) w\right] d x d y .
\end{aligned}
$$

The last term on the right-hand side of (5.14) can be estimated by using (5.5) with $\phi(x)=|x|^{m / 2} \eta_{n}(x)$. Thus,

$$
\begin{gathered}
\left.\left|\int_{\mathbb{R}_{+}^{2}}\right| x\right|^{m / 2} \eta_{n}\left(|x|^{m / 2} \eta_{n}\right)^{\prime} \operatorname{Re}\left[\bar{w}\left(D_{x}+x y\right) w\right] d x d y \mid \\
\leq\left\|\left(D_{x}+x y\right)\left(|x|^{m / 2} \eta_{n} w\right)\right\|\left\|\left(|x|^{m / 2} \eta_{n}\right)^{\prime} w\right\| .
\end{gathered}
$$

From the above and (5.14) we obtain

$$
\begin{aligned}
\left\||x|^{m / 2} \eta_{n} D_{y} w\right\|^{2} \leq & \left(\alpha_{0}+\epsilon \operatorname{Im} \mu_{1}\right)\left\|\left.|| x\right|^{m / 2} \eta_{n} w\right\|^{2}+\operatorname{Im}\left\langle|x|^{m / 2} \eta_{n} w,|x|^{m / 2} \eta_{n} f\right\rangle \\
& +2 \epsilon\left\|\left(D_{x}+x y\right)\left(|x|^{m / 2} \eta_{n} w\right)\right\|\left\|\left(|x|^{m / 2} \eta_{n}\right)^{\prime} w\right\| .
\end{aligned}
$$

The necessary estimate of $\left\|\left(\nabla+i x y \hat{i}_{y}\right) w\right\|$ is obtained by summing up (5.13) and (5.15).

Step 2. Next we show that there exists $c_{1}$, such that if $\phi \in C_{c}^{1}\left(\mathbb{R}_{+}^{2}, \mathbb{C}\right)$, then

$$
\left\||x|^{1 / 2} \phi\right\|^{2} \leq c_{1}\left[\left\|\left(D_{x}+x y\right) \phi\right\|^{2}+\left\|D_{y} \phi\right\|^{2}+\|\phi\|^{2}\right] .
$$

To prove (5.16), we use the same trick as in 4 (line above (2.23)) and write, for $\phi$ with compact support inside $\mathbb{R}_{+}^{2}$, the identity

$$
\int_{\mathbb{R}_{+}^{2}} \frac{x^{2}}{\sqrt{1+x^{2}}}|\phi|^{2} d x d y=-i \int \frac{x}{\sqrt{1+x^{2}}}\left[D_{x}+x y, D_{y}\right] \phi \bar{\phi} d x d y
$$

where $\left[D_{x}+x y, D_{y}\right]$ denotes the commutator of $D_{x}+x y$ and $D_{y}$. Expanding the bracket and after an integration by parts, we obtain

$$
\int_{\mathbb{R}_{+}^{2}} \frac{x^{2}}{\sqrt{1+x^{2}}}|\phi|^{2} d x d y \leq 3 \int_{\mathbb{R}_{+}^{2}}\left(\left|\left(D_{x}+x y\right) \phi\right|^{2}+\left|D_{y} \phi\right|^{2}+\left.\phi\right|^{2}\right) d x d y .
$$

Step 3. Let $(f, w) \in S\left(\overline{\mathbb{R}_{+}^{2}}, \mathbb{C}\right) \times D\left(\mathcal{B}_{\epsilon}\right)$. In this step we claim the existence of a constant $c_{2}$, independent of $(f, w)$, such that for any non-negative real number $m$, and for all $0<\epsilon \leq 1$, we have

$$
\left\||x|^{(m+1) / 2} \eta_{n} w\right\| \leq c_{2}\left[\left\||x|^{m / 2} \eta_{n} w\right\|+\|w\|+\epsilon^{-1}\left\||x|^{m / 2} f\right\|\right] .
$$

Here the cut-off function $\eta_{n}$ is defined by (5.11).

We first note that, since $w \in \tilde{V}$, by approximation, (5.16) holds for

$$
\phi(x, y)=|x|^{m / 2} \eta_{n}(x) w(x, y)
$$

with $m \geq 0$. Consequently, we obtain

$$
\begin{aligned}
\left\||x|^{(m+1) / 2} \eta_{n}(x) w\right\|^{2} \leq c_{1}[ & \left\|\left(D_{x}+x y\right)\left(|x|^{m / 2} \eta_{n}(x) w\right)\right\|^{2} \\
& \left.+\left\|D_{y}\left(|x|^{m / 2} \eta_{n}(x) w\right)\right\|^{2}+\left\||x|^{m / 2} \eta_{n}(x) w\right\|^{2}\right] .
\end{aligned}
$$


We use the Cauchy inequality to control the term

$$
2 \epsilon\left\|\left(D_{x}+x y\right)\left(x^{m / 2} \eta_{n} w\right)\right\|\left\|\left(|x|^{m / 2} \eta_{n}\right)^{\prime} w\right\|
$$

on the right-hand side of (5.15), and then substitute (5.13) and (5.15) into (5.19). We then obtain the existence of $c_{3}>0$, such that

$$
\begin{aligned}
\left\||x|^{(m+1) / 2} \eta_{n} w\right\|^{2} \leq c_{3}[ & \left(\operatorname{Re} \mu_{1}+\alpha_{0}+\epsilon \operatorname{Im} \mu_{1}+1\right)\left\||x|^{m / 2} \eta_{n} w\right\|^{2} \\
& +(1+\epsilon)\left\|\left|\left(|x|^{m / 2} \eta_{n}\right)^{\prime}\right| w\right\|^{2} \\
& +\epsilon^{-1} \operatorname{Re}\left\langle|x|^{m / 2} \eta_{n} w,|x|^{m / 2} \eta_{n} f\right\rangle \\
& \left.+\operatorname{Im}\left\langle|x|^{m / 2} \eta_{n} w,|x|^{m / 2} \eta_{n} f\right\rangle\right] \\
\leq c_{3}[ & \left(\operatorname{Re} \mu_{1}+\alpha_{0}+\epsilon \operatorname{Im} \mu_{1}+3\right)\left\||x|^{m / 2} \eta_{n} w\right\|^{2} \\
& +(1+\epsilon)\left\|\left|\left(|x|^{m / 2} \eta_{n}\right)^{\prime}\right| w\right\|^{2} \\
& \left.+\left(\epsilon^{-2}+1\right)\left\||x|^{m / 2} \eta_{n} f\right\|^{2}\right] .
\end{aligned}
$$

Recall that $\eta_{n}(x)=0$ for $|x| \leq 1$, and that

$$
\left|\eta_{n}^{\prime}(x)\right| \leq \eta_{2 n}(x), \quad|x|^{m / 2} \eta_{n}(x) \leq 1+\eta_{2 n}(x) \quad \text { for }|x| \geq 2 .
$$

Hence

$$
\begin{aligned}
\left\|\left|\left(|x|^{m / 2} \eta_{n}\right)^{\prime}\right| w\right\|^{2} & \leq\left\|\left.\left|\frac{m}{2|x|}\left(|x|^{m / 2} \eta_{n} w\right)\left\|^{2}+\right\|\right| x\right|^{m / 2} \eta_{n}^{\prime}(x) w\right\|^{2} \\
& \leq m^{2}\left\||x|^{m / 2} \eta_{n} w\right\|^{2}+\left\||x|^{m / 2} \eta_{2 n}(x) w\right\|^{2} \\
& \leq\left(2 m^{2}+2\right)\left(\|w\|^{2}+\left\||x|^{m / 2} \eta_{2 n}(x) w\right\|^{2}\right)
\end{aligned}
$$

and

$$
\left\||x|^{m / 2} \eta_{n} w\right\|^{2} \leq 2\|w\|^{2}+2\left\||x|^{m / 2} \eta_{2 n}(x) w\right\|^{2} .
$$

Next, we insert back (5.21) and (5.22) into (5.20), and obtain the existence of $c_{4}$, such that for all $0<\epsilon \leq 1$ we have

$$
\begin{aligned}
\left\||x|^{(m+1) / 2} \eta_{n} w\right\|^{2} \leq & c_{4}\left(1+m^{2}+\alpha_{0}+\left|\mu_{1}\right|\right)\left(\|w\|^{2}+\left\||x|^{m / 2} \eta_{2 n}(x) w\right\|^{2}\right) \\
& +c_{4} \epsilon^{-2}\left\||x|^{m / 2} \eta_{n} f\right\|^{2} .
\end{aligned}
$$

Hence, (5.18) is proved.

Step 4. Proof of (5.9 a). Setting first $m=0$ in (5.18) we obtain

$$
\left\||x|^{1 / 2} \eta_{n} w\right\| \leq c_{2}\left(\left\|\eta_{n} w\right\|+\|w\|+\epsilon^{-1}\|f\|\right) \leq c_{2}\left(2\|w\|+\epsilon^{-1}\|f\|\right) .
$$

Letting $n$ go to $+\infty$ then yields

$$
\left\||x|^{1 / 2} w\right\| \leq c_{2}\left(2\|w\|+\epsilon^{-1}\|f\|\right) .
$$

Consequently, (5.9 $\mathrm{a})$ holds with $k=1 / 2$. For a general half integer $k$, the proof of (5.9 ) now follows by recursively applying the above procedure. 
Step 5. Proof of (5.9 $)$. Let $m \geq 0$ and insert $v(x, y)=y^{m} \eta_{n}^{2}(y) \bar{w}(x, y)$ into (5.10) to obtain

$$
\begin{gathered}
\int_{\mathbb{R}_{+}^{2}}\left\{\epsilon y^{m} \eta_{n}^{2}(y)\left|\left(D_{x}+x y\right) w\right|^{2}+i y^{m} \eta_{n}^{2}(y)\left|D_{y} w\right|^{2}+i\left(y^{m} \eta_{n}^{2}(y)\right)^{\prime} \bar{w} D_{y} w\right. \\
\left.\quad+i y^{m+1} \eta_{n}^{2}(y)|w|^{2}-\lambda y^{m} \eta_{n}^{2}(y)|w|^{2}\right\} d x d y=\int_{\mathbb{R}_{+}^{2}} y^{m} \eta_{n}^{2}(y) f \bar{w} d x d y .
\end{gathered}
$$

From the imaginary part of the above identity and (5.8), we obtain that

$$
\begin{aligned}
\int_{\mathbb{R}_{+}^{2}} & \left\{y^{m} \eta_{n}^{2}(y)\left|D_{y} w\right|^{2}+\left(y^{m} \eta_{n}^{2}(y)\right)^{\prime} \operatorname{Re}\left(\bar{w} D_{y} w\right)+y^{m+1} \eta_{n}^{2}(y)|w|^{2}\right. \\
& \left.-\left(\alpha_{0}+\epsilon \operatorname{Im} \mu_{1}\right) y^{m} \eta_{n}^{2}(y)|w|^{2}\right\} d x d y=\operatorname{Im} \int_{\mathbb{R}_{+}^{2}} y^{m} \eta_{n}^{2}(y) f \bar{w} d x d y .
\end{aligned}
$$

Therefore,

$$
\begin{aligned}
& \left\|y^{m / 2} \eta_{n}(y) D_{y} w\right\|^{2}+\left\|y^{(m+1) / 2} \eta_{n} w\right\|^{2} \\
\leq & \left(\alpha_{0}+\epsilon \operatorname{Im} \mu_{1}\right)\left\|y^{m / 2} \eta_{n}(y) w\right\|^{2}+\left\|\left(y^{m / 2} \eta_{n}(y)\right)^{\prime} w y^{m / 2} \eta_{n}(y) D_{y} w\right\| \\
& +\left|\operatorname{Im}\left\langle\eta_{n}(y) y^{m / 2} w, \eta_{n}(y) y^{m / 2} f\right\rangle\right| \\
\leq & \left(\alpha_{0}+\epsilon \operatorname{Im} \mu_{1}\right)\left\|y^{m / 2} \eta_{n}(y) w\right\|^{2}+\frac{1}{4}\left\|\left(y^{m / 2} \eta_{n}(y)\right)^{\prime} w\right\|^{2}+\left\|y^{m / 2} \eta_{n}(y) D_{y} w\right\|^{2} \\
& +\frac{1}{2}\left\|y^{m / 2} \eta_{n}(y) w\right\|^{2}+\frac{1}{2}\left\|y^{m / 2} \eta_{n}(y) f\right\|^{2} .
\end{aligned}
$$

Hence,

$$
\begin{aligned}
\left\|y^{(m+1) / 2} \eta_{n} w\right\|^{2} \leq & \left(\alpha_{0}+\epsilon \operatorname{Im} \mu_{1}+\frac{1}{2}\right)\left\|y^{m / 2} \eta_{n}(y) w\right\|^{2}+\frac{1}{4}\left\|\left(y^{m / 2} \eta_{n}(y)\right)^{\prime} w\right\|^{2} \\
& +\frac{1}{2}\left\|y^{m / 2} \eta_{n}(y) f\right\|^{2} .
\end{aligned}
$$

Setting $m=0$ in (5.25) we obtain, for sufficiently large $n$, that

$$
\left\|y^{1 / 2} \eta_{n} w\right\|^{2} \leq\left(\alpha_{0}+\epsilon \operatorname{Im} \mu_{1}+1\right)\|w\|^{2}+\|f\|^{2} .
$$

Letting $n \rightarrow+\infty$ in the above inequality yields the existence of $c_{5}$ such that:

$$
\left\|y^{1 / 2} w\right\|^{2} \leq c_{5}\left(\|w\|^{2}+\|f\|^{2}\right)
$$

Invoking inductive arguments we get $(5.9 \mathrm{~b})$ easily.

Step 6. Proof of (5.9k). For a half integer $k \geq 1 / 2$, we use (5.13) with $m=2 k$, and then let $n \rightarrow+\infty$, to obtain the existence of $c_{6}$ such that

$$
\left\|\partial_{x}\left(|x|^{k} w\right)\right\|^{2} \leq c_{6}\left(\left\|\left.|| x\right|^{k} w\right\|^{2}+\|w\|^{2}\right)+\epsilon^{-1}\left\||x|^{k} w\right\|\left\||x|^{k} f\right\|+\left\||x|^{k+1} y w\right\|^{2} .
$$

Combining the above inequality with (5.9 a) and (5.9p) and the inequality (which immediately follows from Young's inequality)

$$
|x|^{p} y^{2} \leq \frac{1}{p+1}\left[p|x|^{p+1}+y^{2 p+2}\right]
$$

completes the proof of (5.95).

In the interest of brevity, we drop from now on the argument involving the cut-off function $\eta_{n}$ (including the limit $n \rightarrow+\infty$ ). 
Step 7. Proof of (5.9d). We differentiate (3.13) with respect to $x$ to obtain

$$
\epsilon\left[\left(D_{x}+x y\right)^{2} \partial_{x} w-2 i y \partial_{x} w+2 x y^{2} w\right]+i\left(D_{y}^{2}+y\right) \partial_{x} w-\lambda \partial_{x} w=\partial_{x} f .
$$

Taking the inner product of (5.27) with $\partial_{x} w$ yields

$$
\begin{aligned}
& \epsilon\left\|\left(D_{x}+x y\right) \partial_{x} w\right\|^{2}-2 i \epsilon\left\langle y \partial_{x} w, \partial_{x} w\right\rangle+2 \epsilon\left\langle x y^{2} w, \partial_{x} w\right\rangle \\
& +i\left\|D_{y} \partial_{x} w\right\|^{2}+i\left\langle y \partial_{x} w, \partial_{x} w\right\rangle-\lambda\left\|\partial_{x} w\right\|^{2}=\left\langle\partial_{x} f, \partial_{x} w\right\rangle .
\end{aligned}
$$

Step 7.1. For the case $k=1 / 2$, the imaginary part of the above identity reads

$$
\begin{aligned}
& \left\|D_{y} \partial_{x} w\right\|^{2}+(1-2 \epsilon)\left\|y^{1 / 2} \partial_{x} w\right\|^{2}+2 \epsilon \operatorname{Im}\left\langle x y^{2} w, \partial_{x} w\right\rangle-\left(\alpha_{0}+\epsilon \operatorname{Im} \mu_{1}\right)\left\|\partial_{x} w\right\|^{2} \\
& \quad=\operatorname{Im}\left\langle\partial_{x} f, \partial_{x} w\right\rangle .
\end{aligned}
$$

Hence, for $0<\epsilon \leq 1 / 4$ we have

$$
\left\|y^{1 / 2} \partial_{x} w\right\|^{2} \leq 4\left[\left(\alpha_{0}+\epsilon \operatorname{Im} \mu_{1}+1\right)\left\|\partial_{x} w\right\|^{2}+\epsilon^{2}\left\|x \partial_{x} w\right\|^{2}+\left\|y^{2} w\right\|^{2}+\left\|\partial_{x} f\right\|^{2}\right] .
$$

We next estimate the first two terms on the right-hand side of the above inequality using (5.9) ) with $k=0$ and $k=1$, respectively. The third term is then estimated using (5.9 $\mathrm{b})$ with $k=2$ to obtain

$$
\left\|y^{1 / 2} \partial_{x} w\right\| \leq c\left[\|w\|+\epsilon^{-1}\left\|\left(|x|^{3 / 2}+y^{3 / 2}+1\right) f\right\|+\left\|\left(|x|^{5 / 2}+y^{3 / 2}+y^{5 / 2}+1\right) f\right\|+\left\|\partial_{x} f\right\|\right] .
$$

Since

$$
\left\||x|^{3 / 2} f\right\| \leq\left\|\left(|x|^{5 / 2}+1\right) f\right\|, \quad\left\|y^{3 / 2} f\right\| \leq\left\|\left(y^{5 / 2}+1\right) f\right\|,
$$

we get

$$
\left\|y^{1 / 2} \partial_{x} w\right\| \leq c\left[\|w\|+\epsilon^{-1}\left\|\left(|x|^{5 / 2}+y^{5 / 2}+1\right) f\right\|+\left\|\partial_{x} f\right\|\right] .
$$

Hence (5.9d) is proved for $k=1 / 2$.

Step 7.2. For the case $k=1$, we take the inner product of (5.27) with $y \partial_{x} w$ to obtain

$$
\begin{aligned}
& \epsilon\left\|y^{1 / 2}\left(D_{x}+x y\right) \partial_{x} w\right\|^{2}-2 i \epsilon\left\|y \partial_{x} w\right\|^{2}+2 \epsilon\left\langle x y^{2} w, y \partial_{x} w\right\rangle+i\left\|y^{1 / 2} D_{y} \partial_{x} w\right\|^{2} \\
& +i\left\langle\partial_{y} \partial_{x} w, \partial_{x} w\right\rangle+i\left\|y \partial_{x} w\right\|^{2}-\lambda\left\langle\partial_{x} w, y \partial_{x} w\right\rangle=\left\langle y^{1 / 2} \partial_{x} f, y^{1 / 2} \partial_{x} w\right\rangle
\end{aligned}
$$

As $w \in D\left(\mathcal{B}_{\epsilon}\right)$ implies $w(x, 0)=0$, we have that $\partial_{x} w(x, 0)=0$. Consequently,

$$
\left\langle\partial_{y} \partial_{x} w, \partial_{x} w\right\rangle=0 .
$$

The imaginary part of (5.29) then reads

$$
\begin{gathered}
\left\|y^{1 / 2} D_{y} \partial_{x} w\right\|^{2}+(1-2 \epsilon)\left\|y \partial_{x} w\right\|^{2}+2 \epsilon \operatorname{Im}\left\langle x y^{2} w, y \partial_{x} w\right\rangle \\
-\left(\alpha_{0}+\epsilon \operatorname{Im} \mu_{1}\right)\left\|y^{1 / 2} \partial_{x} w\right\|^{2}=\operatorname{Im}\left\langle y^{1 / 2} \partial_{x} f, y^{1 / 2} \partial_{x} w\right\rangle .
\end{gathered}
$$

Hence, for $0<\epsilon \leq 1 / 4$, we obtain

$$
\left\|y \partial_{x} w\right\|^{2} \leq 4\left[\left(\alpha_{0}+\epsilon \operatorname{Im} \mu_{1}+1\right)\left\|y^{1 / 2} \partial_{x} w\right\|^{2}+\left\|y^{1 / 2} \partial_{x} f\right\|^{2}+\epsilon^{2}\left\|x \partial_{x} w\right\|^{2}+\left\|y^{3} w\right\|^{2}\right] .
$$


To estimate $\left\|y^{1 / 2} \partial_{x} w\right\|^{2}$ on the right-hand side we may use (5.9 d) for $k=1 / 2$. It seems, however, more transparent to use instead the following inequality:

$$
\begin{aligned}
\int_{\mathbb{R}_{+}^{2}} y^{n-l}\left|\partial_{x} w\right|^{2} d x & \leq \frac{1}{N^{l+1}} \int_{\{y \geq N\}} y^{n+1}\left|\partial_{x} w\right|^{2} d x d y+\int_{\{0 \leq y \leq N\}} y^{n-l}\left|\partial_{x} w\right|^{2} d x d y \\
& \leq \frac{1}{N^{l+1}}\left\|y^{(n+1) / 2} \partial_{x} w\right\|^{2}+N^{n-l}\left\|\partial_{x} w\right\|^{2}
\end{aligned}
$$

which holds for all $N>0$. The above inequality, with $N$ being sufficiently large, $n=1$, and $l=0$, combined together with (5.30), yields

$$
\left\|y \partial_{x} w\right\|^{2} \leq c_{7}\left[\left\|\partial_{x} w\right\|^{2}+\left\|y^{1 / 2} \partial_{x} f\right\|^{2}+\epsilon^{2}\left\|x \partial_{x} w\right\|^{2}+\left\|y^{3} w\right\|^{2}\right] .
$$

Combining the above, (5.9p) (for $k=3$ ), and (5.9) (for $k=0$ and 1) yields the existence of $\epsilon_{0}, c_{8}$ and $c_{9}$, such that for all $\epsilon \in\left(0, \epsilon_{0}\right]$, we have

$$
\begin{aligned}
&\left\|y \partial_{x} w\right\| \leq c_{8}\left[\|w\|+\epsilon^{-1}\left\|\left(|x|^{3 / 2}+y^{3 / 2}+1\right) f\right\|+\left\|y^{1 / 2} \partial_{x} f\right\|\right. \\
&\left.+\epsilon\|w\|+\left\|\left(|x|^{5 / 2}+y^{5 / 2}+1\right) f\right\|+\|w\|+\left\|\left(y^{5 / 2}+1\right) f\right\|\right] \\
& \leq c_{9}\left[\|w\|+\epsilon^{-1}\left\|\left(|x|^{5 / 2}+y^{5 / 2}+1\right) f\right\|+\left\|y^{1 / 2} \partial_{x} f\right\|\right],
\end{aligned}
$$

which completes the proof of $(5.9 \mathrm{~d})$ for $k=1$.

Step 7.3. Finally, we consider the case $k=(m+1) / 2$. Upon taking the inner product of (5.27) with $y^{m} \partial_{x} w$ for an integer $m \geq 1$, we use the identity

$$
\begin{aligned}
i\left\langle D_{y}^{2} \partial_{x} w, y^{m} \partial_{x} w\right\rangle= & i\left\|D_{y}\left(y^{m / 2} \partial_{x} w\right)\right\|^{2}-\frac{i m^{2}}{4}\left\|y^{(m / 2-1)} \partial_{x} w\right\|^{2} \\
& -m \operatorname{Im} \int_{\mathbb{R}_{+}^{2}} y^{m-1} \partial_{y} \partial_{x} w \partial_{x} \bar{w} d x d y
\end{aligned}
$$

to obtain

$$
\begin{aligned}
& \epsilon\left\|y^{m / 2}\left(D_{x}+x y\right) \partial_{x} w\right\|^{2}-2 i \epsilon\left\|y^{(m+1) / 2} \partial_{x} w\right\|^{2} \\
& +2 \epsilon\left\langle y^{m+2} w, x \partial_{x} w\right\rangle+i\left\|D_{y}\left(y^{m / 2} \partial_{x} w\right)\right\|^{2} \\
& -\frac{i m^{2}}{4}\left\|y^{m / 2-1} \partial_{x} w\right\|^{2}-m \operatorname{Im} \int_{\mathbb{R}_{+}^{2}} y^{m-1} \partial_{y} \partial_{x} w \partial_{x} \bar{w} d x d y \\
& +i\left\|y^{(m+1) / 2} \partial_{x} w\right\|^{2}-\lambda\left\|y^{m / 2} \partial_{x} w\right\|^{2} \\
& =\left\langle y^{m / 2} \partial_{x} f, y^{m / 2} \partial_{x} w\right\rangle .
\end{aligned}
$$

The imaginary part of the above is given in the form

$$
\begin{aligned}
& \left\|D_{y}\left(y^{m / 2} \partial_{x} w\right)\right\|^{2}-\frac{m^{2}}{4}\left\|y^{m / 2-1} \partial_{x} w\right\|^{2}+(1-2 \epsilon)\left\|y^{(m+1) / 2} \partial_{x} w\right\|^{2} \\
& +2 \epsilon \operatorname{Im}\left\langle y^{m+2} w, x \partial_{x} w\right\rangle-\left(\alpha_{0}+\epsilon \operatorname{Im} \mu_{1}\right)\left\|y^{m / 2} \partial_{x} w\right\|^{2}=\operatorname{Im}\left\langle y^{m / 2} \partial_{x} f, y^{m / 2} \partial_{x} w\right\rangle .
\end{aligned}
$$

Hence, there exists $c_{10}$, such that, for all $0<\epsilon \leq 1 / 4$, we have

$$
\begin{gathered}
\left\|y^{(m+1) / 2} \partial_{x} w\right\|^{2} \leq c_{10}\left\{\left\|y^{m / 2-1} \partial_{x} w\right\|^{2}+\left\|y^{m+2} w\right\|^{2}+\epsilon^{2}\left\|x \partial_{x} w\right\|^{2}\right. \\
\left.+\left\|y^{m / 2} \partial_{x} w\right\|^{2}+\left\|y^{m / 2} \partial_{x} f\right\|^{2}\right\} .
\end{gathered}
$$


To complete the proof we use (5.31) with $n=m$ twice: with $l=0$ to control $\left\|y^{m} \partial_{x} w\right\|$ and with $l=2$ to control $\left\|y^{m-2} \partial_{x} w\right\|$. For sufficiently large $N$, depending only on $c_{9}$, (5.34) can be converted into the form

$$
\left\|y^{(m+1) / 2} \partial_{x} w\right\|^{2} \leq c_{11}\left[\left\|\partial_{x} w\right\|^{2}+\epsilon^{2}\left\|x \partial_{x} w\right\|^{2}+\left\|y^{m+2} w\right\|^{2}+\left\|y^{m / 2} \partial_{x} f\right\|^{2}\right] .
$$

Using (5.95) with $k=0$ and $k=1$, and (5.9b) with $k=m+2$, we derive from (5.35) the following estimate

$$
\begin{aligned}
\left\|y^{(m+1) / 2} \partial_{x} w\right\| \leq c_{12}[ & \|w\|+\epsilon^{-1}\left\|\left(|x|^{3 / 2}+y^{3 / 2}+1\right) f\right\| \\
& \left.+\left\|\left(|x|^{5 / 2}+y^{5 / 2}+y^{m+3 / 2}\right) f\right\|+\left\|y^{m / 2} \partial_{x} f\right\|\right] .
\end{aligned}
$$

Observing that

$$
\left\||x|^{3 / 2} f\right\| \leq\left\|\left(|x|^{5 / 2}+1\right) f\right\|, \quad\left\|\left(y^{3 / 2}+y^{5 / 2}\right) f\right\| \leq 2\left\|\left(y^{m+3 / 2}+1\right) f\right\|
$$

(we use these inequalities frequently in the sequel without referring to them again), we obtain

$$
\left\|y^{(m+1) / 2} \partial_{x} w\right\| \leq c_{13}\left[\|w\|+\epsilon^{-1}\left\|\left(|x|^{5 / 2}+y^{m+3 / 2}+1\right) f\right\|+\left\|y^{m / 2} \partial_{x} f\right\|\right] .
$$

Thus, (5.9 d) is true for $k=(m+1) / 2$.

Step 8. Proof of (5.9 $)$. The real part of (5.28) reads

$$
\epsilon\left\|\left(D_{x}+x y\right) \partial_{x} w\right\|^{2}+2 \epsilon \operatorname{Re}\left\langle y^{2} w, x \partial_{x} w\right\rangle-\epsilon \operatorname{Re} \mu_{1}\left\|\partial_{x} w\right\|^{2}=\operatorname{Re}\left\langle\partial_{x} f, \partial_{x} w\right\rangle .
$$

Since

$$
\left\|\left(D_{x}+x y\right) \partial_{x} w\right\|^{2}=\left\|\partial_{x x} w\right\|^{2}+\left\|x y \partial_{x} w\right\|^{2}+2 \operatorname{Im}\left\langle x y \partial_{x} w, \partial_{x x} w\right\rangle,
$$

we obtain

$$
\begin{aligned}
\left\|\partial_{x x} w\right\|^{2} & \leq 8\left\|x y \partial_{x} w\right\|^{2}+2\left\|y^{2} w\right\|^{2}+\left(1+2\left|\mu_{1}\right|\right)\left\|\partial_{x} w\right\|^{2}+\epsilon^{-2}\left\|\partial_{x} f\right\|^{2} \\
& \leq 2\left\|y^{2} w\right\|^{2}+\left(1+2\left|\mu_{1}\right|\right)\left\|\partial_{x} w\right\|^{2}+4\left\|x^{2} \partial_{x} w\right\|^{2}+4\left\|y^{2} \partial_{x} w\right\|^{2}+\epsilon^{-2}\left\|\partial_{x} f\right\|^{2} .
\end{aligned}
$$

Using the above, (5.9b) with $k=2$, (5.96) with $k=0$ and $k=2$, and (5.9 d) with $k=2$, we obtain the existence of $c$ and $\epsilon_{0}$ such that

$$
\left\|\partial_{x x} w\right\| \leq c\left[\|w\|+\epsilon^{-1}\left\|\left(|x|^{7 / 2}+y^{7 / 2}+1\right) f\right\|+\left\|y^{3 / 2} \partial_{x} f\right\|+\epsilon^{-1}\left\|\partial_{x} f\right\|\right] .
$$

This verifies (5.9)

It seems worthwhile to note that if one conducts the various computations in the proof of Lemma 5.2 differently, it is possible to get better estimates for (5.9.). However, all these estimates would fit into the general form

$$
\left\|\partial_{x x}^{2} w\right\| \leq C\left[\|w\|+\epsilon^{-1} p(f)\right]
$$

for an appropriately defined norm $p$ on $\mathcal{S}$. 
5.2. Projection on $\left(\operatorname{span}\left\{\psi_{0}\right\}\right)^{\perp}$. We begin by recalling from (2.11) the definition of $\pi_{\|}$, the orthogonal projector on the complex one-dimensional linear subspace generated by $\psi_{0}$. Then, we introduce the complementary projection

$$
\pi_{\perp}=I-\pi_{\|}
$$

where $I$ is the identity map. Making use of topological tensor products, we introduce

$$
\Pi_{\|}=\pi_{\|} \otimes I \quad \text { and } \quad \Pi_{\perp}=\pi_{\perp} \otimes I,
$$

which are orthogonal projectors acting on

$$
L^{2}\left(\mathbb{R}_{+}^{2}, \mathbb{C}\right)=L^{2}\left(\mathbb{R}_{+}, \mathbb{C}\right) \widehat{\otimes} L^{2}(\mathbb{R}, \mathbb{C}) .
$$

For $u$ in $L^{2}\left(\mathbb{R}_{+}^{2}, \mathbb{C}\right)$, we write

$$
u_{\|}=\Pi_{\|} u \quad \text { and } \quad u_{\perp}=\Pi_{\perp} u .
$$

It is easy to show that for all $u \in D(\mathcal{L})$, where $\mathcal{L}$ is the Airy operator defined in (2.10), we have

$$
\alpha_{0}\left\|u_{\|} \mid\right\|^{2}+\alpha_{1}\left\|u_{\perp}\right\|^{2} \leq\langle\mathcal{L} u, u\rangle,
$$

where $\alpha_{0}$ and $\alpha_{1}$ are the lowest and the second eigenvalues of $\mathcal{L}$, and $\alpha_{1}>\alpha_{0}$.

Given $\epsilon>0, \lambda \in \mathcal{C}_{\epsilon, \rho}$ and $f \in \mathcal{S}\left(\overline{\mathbb{R}_{+}^{2}}, \mathbb{C}\right)$, let $w \in D\left(\mathcal{B}_{\epsilon}\right)$ be the solution of (3.13). Write

$$
w=w_{\|}+w_{\perp} .
$$

We next show that for a suitable choice of $\rho, w_{\perp}$ is small, compared with $w_{\|}$, if $\epsilon$ and $f$ are sufficiently small.

Lemma 5.3. There exist positive constants $C, k_{0}$, and $\epsilon_{0}$ such that, for any $\epsilon \in$ $\left(0, \epsilon_{0}\right]$, if $\lambda=\lambda\left(\mu_{1}\right)$ is given by (5.8) and if $(w, f) \in D\left(\mathcal{B}_{\epsilon}\right) \times \mathcal{S}\left(\overline{\mathbb{R}_{+}^{2}}, \mathbb{C}\right)$ satisfies (3.13), then we have

$$
\begin{gathered}
\left\|w_{\perp}\right\|^{2} \leq \frac{1}{\alpha_{1}-\alpha_{0}-2 \epsilon\left|\mu_{1}\right|}\left[\epsilon\left|\mu_{1}\right|\left\|w_{\|}\right\|^{2}+\|w\|\|f\|\right], \\
\left\|x^{2} w_{\perp}\right\|^{2} \leq C\left[\epsilon\|w\|^{2}+\epsilon^{-1}\left\|\left(1+x^{2}\right) f\right\|^{2}\right], \\
\left\|x \partial_{x} w_{\perp}\right\|^{2} \leq C\left[\epsilon\|w\|^{2}+\epsilon^{-1} p_{k_{0}}(f)^{2}\right]
\end{gathered}
$$

where $p_{k_{0}}$ is a norm in $\mathcal{S}\left(\overline{\mathbb{R}_{+}^{2}}, \mathbb{C}\right)$ defined in (3.6).

Proof. Step 1. We prove (5.41a). Let $w$ be the solution of (3.13). Recalling (5.8), we take the inner product of (3.13) with $w$ and then obtain, by considering the imaginary part of the new expression,

$$
\left.\left\langle\left(\mathcal{L}-\mu_{0}\right) w, w\right)\right\rangle=\operatorname{Im}\langle f, w\rangle,
$$

with

$$
\mu_{0}=\operatorname{Im} \lambda=\alpha_{0}+\epsilon \operatorname{Im} \mu_{1} .
$$

It follows from this and (5.39) that

$$
\left(\alpha_{1}-\alpha_{0}-\epsilon \operatorname{Im} \mu_{1}\right)\left\|w_{\perp}\right\|^{2}-\epsilon \operatorname{Im} \mu_{1}\left\|w_{\|}\right\|^{2} \leq\|f\|\|w\|,
$$

from which we deduce $(5.41 \mathrm{k})$ for small $\epsilon$. 
Step 2. We prove (5.41 b). Taking the inner product of (3.13) with $x^{2 k} w$, where $k$ is any positive integer, and computing as in (5.3), we obtain

$$
\begin{aligned}
\left\langle f, x^{2 k} w\right\rangle= & \left\langle\mathcal{B}_{\epsilon} w-\lambda w, x^{2 k} w\right\rangle=\left\langle\epsilon\left(D_{x}+x y\right)^{2} w, w\right\rangle+\left\langle(i \mathcal{L}-\lambda) w, x^{2 k} w\right\rangle \\
= & \epsilon\left\|x^{k}\left(D_{x}+x y\right) w\right\|^{2}+2 k i \epsilon\left\langle\left(D_{x}+x y\right) w, x^{2 k-1} w\right\rangle+\left\langle(i \mathcal{L}-\lambda) w, x^{2 k} w\right\rangle \\
= & \epsilon\left\|x^{k}\left(D_{x}+x y\right) w\right\|^{2}+2 k i \epsilon\left\langle\left(D_{x}+x y\right)\left(x^{k} w\right), x^{k-1} w\right\rangle-2 k^{2} \epsilon\left\|x^{k-1} w\right\|^{2} \\
& +\left\langle(i \mathcal{L}-\lambda)\left(x^{k} w\right), x^{k} w\right\rangle .
\end{aligned}
$$

Combining the imaginary part of the above identity and (5.42) yields

$$
2 k \epsilon \operatorname{Re}\left\langle\left(D_{x}+x y\right)\left(x^{k} w\right), x^{k-1} w\right\rangle+\left\langle\left(\mathcal{L}-\mu_{0}\right)\left(x^{k} w\right), x^{k} w\right\rangle=\operatorname{Im}\left\langle x^{k} f, x^{k} w\right\rangle .
$$

We next make the obvious observation that

$$
\pi_{\|}\left(x^{k} w\right)=x^{k} \pi_{\|} w=x^{k} w_{\|}, \quad \pi_{\perp}\left(x^{k} w\right)=x^{k} \pi_{\perp} w=x^{k} w_{\perp} .
$$

Thus, from (5.39) it follows that

$$
\begin{aligned}
& \alpha_{0}\left\|x^{k} w_{\|}\right\|^{2}+\alpha_{1}\left\|x^{k} w_{\perp}\right\|^{2} \leq\left\langle\mathcal{L}\left(x^{k} w\right), x^{k} w\right\rangle \\
\leq & \mu_{0}\left\|x^{k} w\right\|^{2}+\left\|x^{k} f\right\|\left\|x^{k} w\right\|+2 k \epsilon\left\|\left(D_{x}+x y\right)\left(x^{k} w\right)\right\|\left\|x^{k-1} w\right\| \\
\leq & \mu_{0}\left\|x^{k} w_{\|}\right\|^{2}+\mu_{0}\left\|x^{k} w_{\perp}\right\|^{2}+\frac{1}{2} \epsilon^{-1}\left\|x^{k} f\right\|^{2}+\frac{\epsilon}{2}\left\|x^{k} w\right\|^{2} \\
& +k \epsilon\left\|x^{k-1} w\right\|^{2}+k \epsilon\left\|\left(D_{x}+x y\right)\left(x^{k} w\right)\right\|^{2} .
\end{aligned}
$$

To control the last term on the right-hand side of (5.44), we use (5.13) with $m=2 k$ to obtain the existence of $c_{1}$ for which we have

$$
\begin{aligned}
& k \epsilon \int_{\mathbb{R}_{+}^{2}}\left|\left(D_{x}+x y\right)\left(x^{k} w\right)\right|^{2} d x d y \\
\leq & k \operatorname{Re} \lambda\left\|x^{k} w\right\|^{2}+k \epsilon\left\|\left(x^{k}+k x^{k-1}\right) w\right\|^{2}+k\left\|x^{k} w\right\|\left\|x^{k} f\right\| \\
= & k \epsilon\left(\lambda_{1}+\mu_{1}\right)\left\|x^{k} w\right\|^{2}+2 k \epsilon\left\|x^{k} w\right\|^{2}+2 k^{2} \epsilon\left\|x^{k-1} w\right\|^{2}+\frac{k}{2} \epsilon\left\|x^{k} w\right\|^{2}+\frac{k}{2} \epsilon^{-1}\left\|x^{k} f\right\|^{2} \\
\leq & c_{1} \epsilon\left(\left\|x^{k} w\right\|^{2}+\left\|x^{k-1} w\right\|^{2}\right)+\frac{k}{2} \epsilon^{-1}\left\|x^{k} f\right\|^{2} .
\end{aligned}
$$

Substituting it back into (5.44), we derive the existence of $c_{2}$ such that

$$
\begin{aligned}
& \left(\alpha_{0}-\mu_{0}\right)\left\|x^{k} w_{\|}\right\|^{2}+\left(\alpha_{1}-\mu_{0}\right)\left\|x^{k} w_{\perp}\right\|^{2} \\
& \quad \leq c_{2} \epsilon\left(\left\|x^{k} w\right\|^{2}+\left\|x^{k-1} w\right\|^{2}\right)+c_{2} \epsilon^{-1}\left\|x^{k} f\right\|^{2} .
\end{aligned}
$$

By (5.43) and (5.9k) we then have

$$
\begin{aligned}
& \left(\alpha_{1}-\alpha_{0}\right)\left\|x^{k} w_{\perp}\right\|^{2} \\
\leq & \epsilon \operatorname{Im} \mu_{1}\left(\left\|x^{k} w_{\|}\right\|^{2}+\left\|x^{k} w_{\perp}\right\|^{2}\right)+c_{2} \epsilon\left(\left\|x^{k} w\right\|^{2}+\left\|x^{k-1} w\right\|^{2}\right)+c_{2} \epsilon^{-1}\left\|x^{k} f\right\|^{2} \\
\leq & c_{3} \epsilon\left(\left\|x^{k} w\right\|^{2}+\left\|x^{k-1} w\right\|^{2}\right)+c_{3} \epsilon^{-1}\left\|x^{k} f\right\|^{2} \\
\leq & c_{4} \epsilon\left[\|w\|^{2}+\epsilon^{-2}\left\|\left(|x|^{k-1 / 2}+|x|^{k-3 / 2}+1\right) f\right\|^{2}\right]+c_{2} \epsilon^{-1}\left\|x^{k} f\right\|^{2} \\
= & \left.c_{4} \epsilon\|w\|^{2}+\left(c_{2}+c_{4}\right) \epsilon^{-1} \| x^{k}+|x|^{k-1 / 2}+|x|^{k-3 / 2}+1\right) f \|^{2} .
\end{aligned}
$$

Hence

$$
\left(\alpha_{1}-\alpha_{0}\right)\left\|x^{k} w_{\perp}\right\|^{2} \leq c_{5} \epsilon\|w\|^{2}+c_{5} \epsilon^{-1}\left\|\left(x^{k}+1\right) f\right\|^{2},
$$

which with $k=2$ yields (5.41) ). 
Step 3. Proof of (5.41). We first differentiate (3.13) with respect to $x$ (see also (5.27) ) to obtain

$$
(i \mathcal{L}-\lambda)\left(\partial_{x} w\right)+\epsilon \partial_{x}\left(D_{x}+x y\right)^{2} w=\partial_{x} f .
$$

Then, taking the inner product of the above identity with $x^{2} \partial_{x} w$ yields

$$
\left\langle(i \mathcal{L}-\lambda)\left(x \partial_{x} w\right), x \partial_{x} w\right\rangle+\epsilon\left\langle\partial_{x}\left(D_{x}+x y\right)^{2} w, x^{2} \partial_{x} w\right\rangle=\left\langle\partial_{x} f, x^{2} \partial_{x} w\right\rangle .
$$

The imaginary part, with the aid of (5.43), receives the form

$$
\begin{gathered}
\left\langle\left(\mathcal{L}-\mu_{0}\right)\left(x \partial_{x} w\right), x \partial_{x} w\right\rangle=\operatorname{Im}\left\langle\partial_{x} f, x^{2} \partial_{x} w\right\rangle-\epsilon \operatorname{Im}\left\langle\partial_{x}\left(D_{x}+x y\right)^{2} w, x^{2} \partial_{x} w\right\rangle \\
=\operatorname{Im}\left\langle\partial_{x} f, x^{2} \partial_{x} w\right\rangle+2 \epsilon\left\|x y^{1 / 2} \partial_{x} w\right\|^{2} \\
-\epsilon \operatorname{Im}\left\langle y^{2} w, x^{3} \partial_{x} w\right\rangle+\epsilon \operatorname{Im}\left\langle\left(D_{x}+x y\right)^{2} \partial_{x} w, x^{2} \partial_{x} w\right\rangle .
\end{gathered}
$$

Note that

$\left\langle\left(D_{x}+x y\right)^{2} \partial_{x} w, x^{2} \partial_{x} w\right\rangle=\left\|x\left(D_{x}+x y\right) \partial_{x} w\right\|^{2}+2\left\langle\partial_{x x} w, x \partial_{x} w\right\rangle+2 i\left\|x y^{1 / 2} \partial_{x} w\right\|^{2}$.

Consequently, we have

$$
\begin{aligned}
\left\langle\left(\mathcal{L}-\mu_{0}\right)\left(x \partial_{x} w\right), x \partial_{x} w\right\rangle= & \operatorname{Im}\left\langle\partial_{x} f, x^{2} \partial_{x} w\right\rangle-\epsilon \operatorname{Im}\left\langle y^{2} w, x^{3} \partial_{x} w\right\rangle \\
& +2 \epsilon \operatorname{Im}\left\langle\partial_{x x} w, x \partial_{x} w\right\rangle+2 \epsilon\left\|x y^{1 / 2} \partial_{x} w\right\|^{2} .
\end{aligned}
$$

Combining the above with (5.39) we obtain

$$
\begin{aligned}
& \left(\alpha_{0}-\mu_{0}\right)\left\|x \partial_{x} w_{\|}\right\|^{2}+\left(\alpha_{1}-\mu_{0}\right)\left\|x \partial_{x} w_{\perp}\right\|^{2} \\
\leq & \left\langle\left(\mathcal{L}-\mu_{0}\right)\left(x \partial_{x} w\right), x \partial_{x} w\right\rangle \\
\leq & \left\|\partial_{x} f\right\|\left\|x^{2} \partial_{x} w\right\|+\epsilon\left\|y^{2} w\right\|\left\|x^{3} \partial_{x} w\right\|+2 \epsilon\left|\left\langle\partial_{x x} w, x \partial_{x} w\right\rangle\right|+2 \epsilon\left\|x y^{1 / 2} \partial_{x} w\right\|^{2},
\end{aligned}
$$

which together with (5.43) yields

$$
\begin{gathered}
\left(\alpha_{1}-\mu_{0}\right)\left\|x \partial_{x} w_{\perp}\right\|^{2} \\
\leq C \epsilon\left[\left\|x^{2} \partial_{x} w_{\|}\right\|^{2}+\left\|x \partial_{x} w\right\|^{2}+\left\|y^{2} w\right\|^{2}+\left\|x^{3} \partial_{x} w\right\|^{2}\right. \\
\left.\quad+\left\|\left\langle\partial_{x x} w, x \partial_{x} w\right\rangle \mid+\right\| x y^{1 / 2} \partial_{x} w \|^{2}\right]+\epsilon^{-1}\left\|\partial_{x} f\right\|^{2} \\
\leq C \epsilon\left[\left\|y^{2} w\right\|^{2}+\left\|x \partial_{x} w\right\|^{2}+\left\|x^{2} \partial_{x} w\right\|^{2}\right. \\
\left.+\left\|x^{3} \partial_{x} w\right\|^{2}+\left\|y \partial_{x} w\right\|^{2}+\left\|\partial_{x x} w\right\|^{2}\right]+\epsilon^{-1}\left\|x \partial_{x} f\right\|^{2} .
\end{gathered}
$$

Using (5.9b) with $k=1$, (5.9k) with $k=1,2,3$, (5.9d) with $k=1$, and (5.9k), we obtain for all $0<\epsilon \leq 1 / 4$ that

$$
\begin{aligned}
& \left(\alpha_{1}-\mu_{0}\right)\left\|x \partial_{x} w_{\perp}\right\|^{2} \\
& \leq C \epsilon\left\{\|w\|^{2}+\epsilon^{-2}\left\|\left(1+|x|^{9 / 2}+y^{9 / 2}\right) f\right\|^{2}\right. \\
& \left.+\left\|\left(y^{1 / 2}+y^{3 / 2}\right) \partial_{x} f\right\|^{2}+\epsilon^{-2}\left\|\partial_{x} f\right\|^{2}\right\}+\epsilon^{-1}\left\|x \partial_{x} f\right\|^{2} .
\end{aligned}
$$

Hence (5.41) is verified for $0<\epsilon \leq 1 / 4$, with

$$
p(f)^{2}=\left\|\left(1+|x|^{9 / 2}+y^{9 / 2}\right) f\right\|^{2}+\left\|\left(1+|x|+y^{3 / 2}\right) \partial_{x} f\right\|^{2} .
$$

We are now ready for the final stage of Step 4 of the proof for the main results stated in Section 3, in which we obtain an estimate of the norm of the solution $w=\left(\mathcal{B}_{\epsilon}-\lambda\right)^{-1} f$ of (3.13) for $\lambda \in \mathcal{C}_{\epsilon, \rho}$ and $f \in \mathcal{S}$. Recall that every $\lambda$ on the circle $\mathcal{C}_{\epsilon, \rho}$ obeys the form

$$
\lambda=\lambda(\delta)=\gamma_{\epsilon}+\epsilon \delta(\rho, \phi, \epsilon)
$$


where $\gamma_{\epsilon}$ is given in (3.4), and

$$
\delta(\rho, \phi, \epsilon):=\rho \exp (i \phi) \epsilon^{1 / 2}, \quad \phi \in[0,2 \pi) .
$$

Lemma 5.4. There exist $k_{0} \in \mathbb{N}, \rho_{0}>0$, and $C>0$, such that for any $w$ satisfying (3.13) with $\lambda \in C_{\epsilon, \rho}$ and $\rho>\rho_{0}$ it holds that

$$
\exists \epsilon_{0}(\rho): \quad 0<\epsilon<\epsilon_{0}(\rho) \Longrightarrow\|w\| \leq C(\epsilon|\delta|)^{-1} p_{k_{0}}(f),
$$

where $p_{k_{0}}$ is the norm in $\mathcal{S}\left(\overline{\mathbb{R}_{+}^{2}}, \mathbb{C}\right)$ given in Lemma 5.3 .

Proof. Step 1. Let

$$
w_{1}(x)=\int_{0}^{+\infty} w(x, y) \psi_{0}(y) d y
$$

Then,

$$
w_{\|}(x, y)=w_{1}(x) \psi_{0}(y), \quad\left\|w_{\|}\right\|=\left\|w_{1}\right\|_{L^{2}(\mathbb{R})} .
$$

Taking the inner product of (3.13) with $\psi_{0}$ in $L^{2}\left(\mathbb{R}_{+}, \mathbb{C}\right)$ with respect to the variable $y$ and dividing the resulting equation by $\epsilon$, we obtain

$$
D_{x}^{2} w_{1}-2 i x \partial_{x}\left\langle w, y \psi_{0}\right\rangle_{y}-i\left\langle w, y \psi_{0}\right\rangle_{y}+x^{2}\left\langle w, y^{2} \psi_{0}\right\rangle_{y}-\frac{\lambda-i \alpha_{0}}{\epsilon} w_{1}=\frac{1}{\epsilon}\langle f, w\rangle_{y},
$$

where $\langle\cdot, \cdot\rangle_{y}$ denotes the inner product in $L^{2}\left(\mathbb{R}_{+}, \mathbb{C}\right)$. Having in mind the definition (4.9) - 4.10) of the operator $\mathcal{K}_{0}$, we can rewrite $D_{x}^{2} \phi$, for any smooth $\phi$, in the form

$$
D_{x}^{2} \phi=\mathcal{K}_{0} \phi+2 i \alpha x \partial_{x} \phi+i \alpha \phi-\beta^{2} x^{2} \phi
$$

Therefore,

$$
\begin{aligned}
& D_{x}^{2} w_{1}-2 i x \partial_{x}\left\langle w, y \psi_{0}\right\rangle_{y}-i\left\langle w, y \psi_{0}\right\rangle_{y}+x^{2}\left\langle w, y^{2} \psi_{0}\right\rangle_{y} \\
= & \mathcal{K}_{0} w_{1}-2 i x \partial_{x}\left[\left\langle w, y \psi_{0}\right\rangle_{y}-\alpha w_{1}\right]-i\left[\left\langle w, y \psi_{0}\right\rangle_{y}-\alpha w_{1}\right]+x^{2}\left[\left\langle w, y^{2} \psi_{0}\right\rangle_{y}-\beta^{2} w_{1}\right] \\
= & \mathcal{K}_{0} w_{1}-2 i x \partial_{x}\left\langle w_{\perp}, y \psi_{0}\right\rangle_{y}-i\left\langle w_{\perp}, y \psi_{0}\right\rangle_{y}+x^{2}\left\langle w_{\perp}, y^{2} \psi_{0}\right\rangle_{y}
\end{aligned}
$$

where we have used the identities

$$
\begin{aligned}
& \left\langle w, y \psi_{0}\right\rangle_{y}-\alpha w_{1}=\int_{0}^{+\infty} y \psi_{0}\left(w-w_{1}(x) \psi_{0}(y)\right) d y=\left\langle w-w_{\|}, y \psi_{0}\right\rangle_{y}=\left\langle w_{\perp}, y \psi_{0}\right\rangle_{y}, \\
& \left\langle w, y^{2} \psi_{0}\right\rangle_{y}-\beta^{2} w_{1}=\int_{0}^{+\infty} y^{2} \psi_{0}\left(w-w_{1} \psi_{0}\right) d y=\left\langle w-w_{\|}, y^{2} \psi_{0}\right\rangle_{y}=\left\langle w_{\perp}, y^{2} \psi_{0}\right\rangle_{y} .
\end{aligned}
$$

We then combine (5.49) and (5.51), using (5.46) and (5.47) to get

$$
\left(\mathcal{K}_{0}-\lambda_{1}-\delta\right) w_{1}=g,
$$

where

$$
g=2 i x\left\langle\partial_{x} w_{\perp}, y \psi_{0}\right\rangle_{y}+i\left\langle w_{\perp}, y \psi_{0}\right\rangle_{y}-x^{2}\left\langle w_{\perp}, y^{2} \psi_{0}\right\rangle_{y}+\epsilon^{-1}\left\langle f, \psi_{0}\right\rangle_{y}
$$

Step 2. We next estimate $\|g\|$. Since

$$
\begin{aligned}
\left\|x\left\langle\partial_{x} w_{\perp}, y \psi_{0}\right\rangle_{y}\right\|_{L^{2}(\mathbb{R})}^{2} & \leq\|x\| y \psi_{0}\left\|_{L^{2}\left(\mathbb{R}_{+}\right)}\right\| \partial_{x} w_{\perp}\left\|_{L^{2}\left(\mathbb{R}_{+}\right)}\right\|_{L^{2}(\mathbb{R})}^{2} \\
& \leq\left\|y \psi_{0}\right\|_{L^{2}\left(\mathbb{R}_{+}\right)}^{2}\left\|x \partial_{x} w_{\perp}\right\|^{2} \leq C\left\|x \partial_{x} w_{\perp}\right\|^{2},
\end{aligned}
$$

and since there exists $C>0$ such that

$$
\left\|x^{2}\left\langle y^{2} \psi_{0}, w_{\perp}\right\rangle_{y}\right\|_{L^{2}(\mathbb{R})}^{2} \leq C\left\|x^{2} w_{\perp}\right\|^{2},
$$


we have

$$
\|g\|_{L^{2}(\mathbb{R})}^{2} \leq C\left[\left\|x \partial_{x} w_{\perp}\right\|^{2}+\left\|w_{\perp}\right\|^{2}+\left\|x^{2} w_{\perp}\right\|^{2}+\epsilon^{-2}\|f\|^{2}\right] .
$$

Consequently, by (5.41) we have, for some $k_{0}$,

$$
\|g\|_{L^{2}(\mathbb{R})}^{2} \leq C\left[\epsilon\|w\|^{2}+\epsilon^{-2} p_{k_{0}}(f)^{2}\right] .
$$

Step 3. As the operator $\mathcal{K}_{0}$ is self-adjoint we obtain from (5.52) and the spectral theorem [16] that for any fixed $\rho>0$ there exists $\epsilon_{0}(\rho)>0$ such that for all $0<\epsilon<\epsilon_{0}(\rho)$ we have

$$
\left\|w_{1}\right\|_{L^{2}(\mathbb{R})} \leq 2 \frac{\|g\|_{L^{2}(\mathbb{R})}}{|\delta|} .
$$

Substituting (5.54) into (5.55), we obtain for some $k_{0} \in \mathbb{N}$,

$$
\left\|w_{1}\right\|_{L^{2}(\mathbb{R})} \leq C_{1}|\delta|^{-1}\left[\epsilon^{1 / 2}\|w\|+\epsilon^{-1} p_{k_{0}}(f)\right] .
$$

By (5.41 a), there exists $C_{2}>0$ such that, for small $\epsilon$,

$$
\|w\|^{2} \leq C_{2}\left[\left\|w_{\|}\right\|^{2}+\|w\|\|f\|\right],
$$

and hence, noting that $\left\|w_{1}\right\|_{L^{2}(\mathbb{R})}=\left\|w_{\|}\right\|$, we have that

$$
\left\|w_{1}\right\|_{L^{2}(\mathbb{R})}^{2} \geq \frac{1}{2 C_{2}}\left[\|w\|^{2}-C_{2}^{2}\|f\|^{2}\right] .
$$

Using the above inequality together with (5.56), we obtain the existence of $k_{0}$ and $C_{3}$ such that

$$
\|w\|^{2} \leq C_{2}^{2}\|f\|^{2}+2 C_{2}\left\|w_{1}\right\|_{L^{2}(\mathbb{R})}^{2} \leq C_{3} \delta^{-2}\left[\epsilon\|w\|^{2}+\epsilon^{-2} p_{k_{0}}(f)^{2}\right],
$$

which is valid for all $\epsilon \in\left(0, \epsilon_{0}(\rho)\right)$.

Then let

$$
\rho_{0}=\sqrt{2 C_{3}} .
$$

For a given $\rho>\rho_{0}$, we obtain from (5.58) that for all $\epsilon \in\left(0, \epsilon_{0}(\rho)\right)$ there exist $k_{0} \in \mathbb{N}$ and $C_{4}>0$ such that

$$
\|w\| \leq C_{4}(\epsilon \delta)^{-1} p_{k_{0}}(f) .
$$

As an immediate consequence of Lemma 5.4, we obtain

Proposition 5.5. Let $w$ satisfy (3.13) with $f \equiv 0$ and $\lambda \in \mathcal{C}_{\epsilon, \rho}$, where $\epsilon$ is small enough. Then $w \equiv 0$.

Proof of Proposition 3.4. (3.11) follows from (5.48) with $|\delta|=\rho \epsilon^{1 / 2}$.

The other consequences of Lemma 5.4 have been described at the end of Section 3 


\section{Existence, UPPER AND LOWER BOUNDS}

It is now possible to state the following existence result:

Proposition 6.1. Let

$$
\gamma_{\epsilon}=i \alpha_{0}+\epsilon \lambda_{1}
$$

where $\alpha_{0}$ is the first eigenvalue of the Airy operator $\mathcal{L}$ and $\lambda_{1}$ is the first eigenvalue of the operator $\mathcal{K}_{0}$ defined in (4.9). Then there exist $\epsilon_{0}>0$ and $C>0$ such that, for all $0<\epsilon<\epsilon_{0}$,

$$
\sigma\left(\mathcal{B}_{\epsilon}\right) \cap B\left(\gamma_{\epsilon}, C \epsilon^{3 / 2}\right) \neq \emptyset .
$$

Proof. The proof follows immediately from (3.10) and (5.48).

By (5.48) it follows that $\mathcal{B}_{\epsilon}-\lambda$ is injective for $\lambda \in \mathcal{C}(\epsilon, \rho)$ with $\epsilon$ small enough. As $\mathcal{B}_{\epsilon}$ has a compact resolvent it immediately follows that $\lambda \notin \sigma\left(\mathcal{B}_{\epsilon}\right)$.

Coming back to our initial operator $\mathcal{A}_{c}^{+}$, we deduce from Proposition 6.1 the following corollary.

Corollary 6.2. The operator $\mathcal{A}_{c}^{+}$defined in the introduction admits at least one eigenvalue $\mu=\mu(c)$ having the following expansion as $|c| \rightarrow+\infty$ :

$$
\mu(c)=c^{2 / 3} \exp \left(i \frac{\pi}{3}\right) \alpha_{0}+\lambda_{1} \exp \left(-i \frac{\pi}{6}\right) c^{-1 / 3}+\mathcal{O}\left(c^{-5 / 6}\right) .
$$

Clearly, (6.3) establishes the upper bound in (1.18). To obtain the lower bound we first need to prove Proposition 3.5.

Proof of Proposition 3.5. The resolvent of $\mathcal{B}_{\epsilon}$ being compact, it is sufficient to show that there are no eigenvalues in $D_{b, \epsilon}$. Then let $\lambda \in D_{b, \epsilon}$, where $D_{b, \epsilon}$ is given by (3.14), and let $w \in D\left(\mathcal{B}_{\epsilon}\right)$ satisfy

$$
\left(\mathcal{B}_{\epsilon}-\lambda\right) w=0 .
$$

We note first that, for fixed $b$ and sufficiently small $\epsilon>0$, both (5.9) and (5.41) hold for $\lambda \in D_{b, \epsilon}$. Furthermore, despite the fact that Lemma 5.4 is stated for $\lambda \in \mathcal{C}_{\epsilon, \rho}$ (see (5.46), (5.47)), we can still use (5.54), (5.57), and even (5.52) as long as we appropriately modify the definition of $\delta$. More precisely, $\lambda=\gamma_{\epsilon}+\epsilon \delta \in D_{b, \epsilon}$ means that instead of (5.47) we have

$$
-\lambda_{1}<\operatorname{Re} \delta \leq-b \epsilon^{1 / 2}, \quad 0<\operatorname{Im} \delta \leq 3 \lambda_{1} .
$$

We can thus take the inner product with respect to $x$, in $L^{2}(\mathbb{R}, \mathbb{C})$, of $(5.52)$ with $w_{1}$, defined in the proof of Lemma [5.4, to obtain

$$
\begin{aligned}
& -\operatorname{Re} \delta\left\|w_{1}\right\|_{L^{2}(\mathbb{R})}^{2} \leq \operatorname{Re}\left\langle\left(\mathcal{K}_{0}-\lambda_{1}\right) w_{1}, w_{1}\right\rangle_{x}-\operatorname{Re} \delta\left\|w_{1}\right\|_{L^{2}(\mathbb{R})}^{2} \\
& \quad=\left\langle g, w_{1}\right\rangle_{x} \leq\|g\|_{L^{2}(\mathbb{R})}\left\|w_{1}\right\|_{L^{2}(\mathbb{R})},
\end{aligned}
$$

where $g$ was defined after (5.52). By (5.54), with $f=0$, it then follows that

$$
b \epsilon^{1 / 2}\left\|w_{1}\right\|_{L^{2}(\mathbb{R})} \leq C \epsilon^{1 / 2}\|w\| .
$$

Using (5.57) with $f=0$ then yields

$$
\frac{b}{\sqrt{2 C_{2}}}\|w\| \leq C\|w\| .
$$

For sufficiently large $b$ we must then have $w \equiv 0$, which means that $\lambda$ is not an eigenvalue. 


\section{DECAY OF THE SEMI-GROUP}

In this section we apply a technique which was first applied in [13] to obtain stability of the normal states for the linearized one-dimensional Ginzburg-Landau system, in the absence of any magnetic field (induced or applied). The linear operator in this case is the Dirichlet realization of the complex Airy operator in $\mathbb{R}_{+}$ 14, which we denote here by $\mathcal{Q}$. In 3, it has been shown that when $\lambda$ lies to the left of $\sigma(\mathcal{Q})$, then

$$
\left\|e^{-t(\mathcal{Q}-\lambda)} u_{0}\right\|_{L^{2}\left(\mathbb{R}_{+}\right) \underset{t \rightarrow+\infty}{\longrightarrow} 0} 0
$$

when $u_{0}$ belongs to a certain dense set in $L^{2}\left(\mathbb{R}_{+}\right)$. In 13 the validity of the above has been extended to any $u_{0} \in L^{2}\left(\mathbb{R}_{+}\right)$. We use the technique in [13] to obtain a similar result, but this time for the operator $\mathcal{A}_{c}^{+}$, i.e., we prove Theorem 1.3. This desired outcome is a consequence of the following proposition.

Let $\mathcal{P}$ be the operator defined in (2.1), and let

$$
G(t)=\exp (-t \mathcal{P})
$$

be the semigroup whose generator is $\mathcal{P}$. Let $\mu_{m}$ be the number defined in (1.17).

Proposition 7.1. For any $\omega$ satisfying

$$
\omega<\mu_{m},
$$

there exists $M_{\omega}>0$ such that

$$
\|G(t)\|_{\mathcal{L}\left(L^{2}\left(\mathbb{R}_{+}^{2}, \mathbb{C}\right)\right)} \leq M_{\omega} \exp (-\omega t) .
$$

Furthermore, for any $\omega>\mu_{m}$ we have that

$$
\left\|e^{\omega t} G(t)\right\|_{\mathcal{L}\left(L^{2}\left(\mathbb{R}_{+}^{2}, \mathbb{C}\right)\right)} \geq \exp \left\{\left(\omega-\mu_{m}\right) t\right\} .
$$

Note that by (2.9) we have that

$$
\inf _{z \in \sigma\left(\mathcal{A}_{c}^{+}\right)} \operatorname{Re} z \geq \frac{1}{2}|c|^{2 / 3} \alpha_{0} .
$$

Furthermore, for a sufficiently large value of $c$, in view of (1.18), we can approximate $\mu_{m}$ using (6.3). In the unlikely event that for some value of $c$, the spectrum of $\mathcal{A}_{c}^{+}$is empty we may set $\mu_{m}=+\infty$, i.e., (7.2) would hold for all $\omega \in \mathbb{R}$, and (7.3) would become redundant.

We prove (7.2) with $\mathcal{A}_{c}^{+}$replaced by $\mathcal{P}$ defined in (2.1) since they are unitarily equivalent by (2.1). In order to make use of the Gearhart-Prüss Theorem (see the Appendix) we need the following lemma.

Lemma 7.2. Let $\mu_{m}$ be the number defined in (17.1). For any real number $\alpha$ satisfying $\alpha<\mu_{m}$, there exists a constant $C$, such that for all $\lambda$ with $\operatorname{Re} \lambda \leq \alpha$, we have

$$
\left\|(\mathcal{P}-\lambda)^{-1}\right\| \leq C
$$

Proof. Without loss of generality we assume $\alpha>0$. Then let $\lambda$ be such that $\operatorname{Re} \lambda<\alpha$. Clearly, $\lambda$ lies outside the spectrum of $\mathcal{A}_{c}^{+}$, and hence outside the spectrum of $\mathcal{P}$ as well. Consequently, once we manage to prove boundedness of the resolvent $(\mathcal{P}-\lambda)^{-1}$ as $|\lambda| \rightarrow+\infty$ (for $\operatorname{Re} \lambda<\alpha$ ), then the lemma will be proved. 
We distinguish between three different subdomains of $\mathbb{C}$ :

(1) $\operatorname{Re} \lambda \leq-\alpha$.

(2) $\operatorname{Im} \lambda \rightarrow-\infty$, and $\operatorname{Re} \lambda \in(-\alpha, \alpha)$.

(3) $\operatorname{Im} \lambda \rightarrow+\infty$, and $\operatorname{Re} \lambda \in(-\alpha, \alpha)$.

In the first region we use the inequality

(7.5) $\operatorname{Re}\langle(\mathcal{P}-\lambda) u, u\rangle=\left\|\left(D_{x}+y x\right) u\right\|^{2}+\left\|D_{y} u\right\|^{2}-\operatorname{Re} \lambda\|u\|^{2} \geq\left(E_{0}^{*}-\operatorname{Re} \lambda\right)\|u\|^{2}$, where $E_{0}^{*}$ was given in (1.14), to obtain that

$$
\left\|(\mathcal{P}-\lambda)^{-1}\right\| \leq \frac{1}{E_{0}^{*}+\alpha} .
$$

In the second case we use the inequality

$$
\operatorname{Im}\langle(\mathcal{P}-\lambda) u, u\rangle=\int_{\mathbb{R}_{+}^{2}}(y-\operatorname{Im} \lambda)|u|^{2} \geq-\operatorname{Im} \lambda\|u\|^{2},
$$

yielding immediately that

$$
\left\|(\mathcal{P}-\lambda)^{-1}\right\| \leq \frac{1}{|\operatorname{Im} \lambda|} .
$$

Consequently, it remains necessary to control the norm of the resolvent only in the third case.

Next, we prove the lemma in the third case. To this end, we borrow ideas from semi-classical analysis. The main idea of the proof is to approximate $(\mathcal{P}-\lambda)^{-1}$ by a sum of two different operators: one of them should serve as a good approximation when applied to functions supported near the boundary, while the other one should take care of functions whose support lies far away from the boundary.

The first component in the above decomposition is thus derived from the Dirichlet realization of the differential operator

$$
\left(D_{x}+y x\right)^{2}+D_{y}^{2}+i(y-\operatorname{Im} \lambda)-\operatorname{Re} \lambda,
$$

for $(x, y)$ in the band

$$
\mathcal{S}=\mathbb{R} \times\left(0, \frac{\operatorname{Im} \lambda}{2}\right) .
$$

Denote the resolvent of this operator by

$$
\mathcal{R}_{1}(\lambda): L^{2}(\mathcal{S}, \mathbb{C}) \rightarrow H_{\text {mag }}^{1}(\mathcal{S}, \mathbb{C}) .
$$

Since (7.6) still holds for the above Dirichlet realization, we easily obtain that

$$
\left\|\mathcal{R}_{1}(\lambda)\right\| \leq \frac{2}{\operatorname{Im} \lambda} .
$$

Furthermore, by (7.5), which remains valid for the problem in $\mathcal{S}$, we have for $u=\mathcal{R}_{1} f$

$$
\begin{gathered}
\left\|D_{y} \mathcal{R}_{1}(\lambda) f\right\|^{2}=\left\|D_{y} u\right\|^{2} \leq\|(\mathcal{P}-\lambda) u\|\|u\|+\operatorname{Re} \lambda\|u\|^{2} \\
\leq\|f\|\left\|\mathcal{R}_{1}(\lambda) f\right\|+|\alpha|\left\|\mathcal{R}_{1}(\lambda) f\right\|^{2} \leq\left(\frac{2}{\operatorname{Im}|\lambda|}+\frac{4|\alpha|}{|\operatorname{Im} \lambda|^{2}}\right)\|f\|^{2} .
\end{gathered}
$$

Hence

$$
\left\|D_{y} \mathcal{R}_{1}(\lambda)\right\| \leq\left[\frac{2}{|\operatorname{Im} \lambda|}\right]^{1 / 2}+\frac{2 \alpha^{1 / 2}}{|\operatorname{Im} \lambda|}
$$


Far from the boundary, we attempt to approximate the resolvent of the same differential operator on $\mathbb{R}^{2}$, neglecting the boundary effect. Denote this resolvent by $\mathcal{R}_{2}(\lambda)$. Recall from 4 that the norm $\left\|\mathcal{R}_{2}(\lambda)\right\|$ is independent of $\operatorname{Im} \lambda$ because the problem in $\mathbb{R}^{2}$ is invariant to translations in $y$ variable. Since $\mathcal{R}_{2}(\lambda)$ is an entire function in $\lambda$, we easily obtain a uniform bound on $\left\|\mathcal{R}_{2}(\lambda)\right\|$ for $\operatorname{Re} \lambda \in[-\alpha,+\alpha]$. Hence,

$$
\left\|\mathcal{R}_{2}(\lambda)\right\| \leq C_{1}(\alpha), \quad\left\|D_{y} \mathcal{R}_{2}(\lambda)\right\| \leq C(\alpha) .
$$

We now use a partition of unity in the $y$ variable in order to construct an approximate inverse $\mathcal{R}^{\mathrm{app}}(\lambda)$ for $\mathcal{P}-\lambda$. We shall then prove that the difference between the approximation and the exact resolvent is well controlled as $\operatorname{Im} \lambda \rightarrow+\infty$. Define then the pair of cutoff functions in $C^{\infty}\left(\mathbb{R}_{+},[0,1]\right)$,

$$
\begin{aligned}
& \phi(t)=0 \text { on }[0,1 / 4], \quad \phi(t)=1 \text { on }[1 / 2,+\infty), \\
& \psi(t)=\sqrt{1-\phi(t)^{2}} \text { on } \mathbb{R}_{+} .
\end{aligned}
$$

and then set

$$
\phi_{\lambda}(y)=\phi\left(\frac{y}{\operatorname{Im} \lambda}\right), \quad \psi_{\lambda}(y)=\psi\left(\frac{y}{\operatorname{Im} \lambda}\right) .
$$

The approximate inverse $\mathcal{R}^{\mathrm{app}}(\lambda)$ is then constructed as

$$
\mathcal{R}^{\mathrm{app}}(\lambda)=\psi_{\lambda} \mathcal{R}_{1}(\lambda) \psi_{\lambda}+\phi_{\lambda} \mathcal{R}_{2}(\lambda) \phi_{\lambda},
$$

where $\phi_{\lambda}$ and $\psi_{\lambda}$ denote the operators of multiplication by the functions $\phi_{\lambda}(y)$ and $\psi_{\lambda}(y)$. Thus,

$$
\begin{aligned}
& \psi_{\lambda}: D\left(\mathcal{R}_{1}^{-1}\right) \rightarrow D(\mathcal{P}), \\
& \phi_{\lambda}: D\left(\mathcal{R}_{2}^{-1}\right) \rightarrow D(\mathcal{P}) .
\end{aligned}
$$

From (7.7) and (7.9) we get, for sufficiently large $\operatorname{Im} \lambda$,

$$
\left\|\mathcal{R}^{\text {app }}(\lambda)\right\| \leq C_{3}(\alpha) \text {. }
$$

Note that

$$
\left|\psi_{\lambda}^{\prime}(y)\right| \leq \frac{C}{|\operatorname{Im} \lambda|}, \quad\left|\psi_{\lambda}^{\prime \prime}(y)\right| \leq \frac{C}{|\operatorname{Im} \lambda|^{2}} .
$$

and that

$$
\left|\phi_{\lambda}^{\prime}(y)\right| \leq \frac{C}{|\operatorname{Im} \lambda|}, \quad\left|\phi_{\lambda}^{\prime \prime}(y)\right| \leq \frac{C}{|\operatorname{Im} \lambda|^{2}} .
$$

Next, we apply $\mathcal{P}-\lambda$ to $\mathcal{R}^{\text {app }}$ to obtain that

$$
(\mathcal{P}-\lambda) \mathcal{R}^{\mathrm{app}}(\lambda)=I+\left[\mathcal{P}, \psi_{\lambda}\right] \mathcal{R}_{1}(\lambda) \psi_{\lambda}+\left[\mathcal{P}, \phi_{\lambda}\right] \mathcal{R}_{2}(\lambda) \phi_{\lambda},
$$

where $I$ is the identity operator, and

$$
\left[\mathcal{P}, \phi_{\lambda}\right] \equiv \mathcal{P} \phi_{\lambda}-\phi_{\lambda} \mathcal{P}=\left[D_{y}^{2}, \phi_{\lambda}\right]=-\frac{2 i}{\operatorname{Im} \lambda} \phi^{\prime}\left(\frac{y}{\operatorname{Im} \lambda}\right) D_{y}-\frac{1}{(\operatorname{Im} \lambda)^{2}} \phi^{\prime \prime}\left(\frac{y}{\operatorname{Im} \lambda}\right) .
$$

A similar relation holds for $\left[\mathcal{P}, \psi_{\lambda}\right]$. Here we have used (17.10) and the fact that

$$
(\mathcal{P}-\lambda) \mathcal{R}_{1}(\lambda) \psi_{\lambda} u=\psi_{\lambda} u, \quad(\mathcal{P}-\lambda) \mathcal{R}_{2}(\lambda) \phi_{\lambda} u=\phi_{\lambda} u, \quad \forall u \in L^{2}\left(\mathbb{R}_{+}^{2}, \mathbb{C}\right) .
$$

Using (7.7), (7.8), (7.9), and (7.15), we then easily obtain, for $\operatorname{Im} \lambda$ large enough,

$$
\left\|\left[\mathcal{P}, \psi_{\lambda}\right] \mathcal{R}_{1}(\lambda)\right\|+\left\|\left[\mathcal{P}, \phi_{\lambda}\right] \mathcal{R}_{2}(\lambda)\right\| \leq \frac{C_{4}(\alpha)}{|\operatorname{Im} \lambda|} .
$$


Hence, if $|\operatorname{Im} \lambda|$ is sufficiently large, then $I+\left[\mathcal{P}, \psi_{\lambda}\right] \mathcal{R}_{1}(\lambda) \psi_{\lambda}+\left[\mathcal{P}, \phi_{\lambda}\right] \mathcal{R}_{2}(\lambda) \phi_{\lambda}$ is invertible in $\mathcal{L}\left(L^{2}\left(\mathbb{R}_{+}^{2}, \mathbb{C}\right)\right)$, and

$$
\left\|\left(I+\left[\mathcal{P}, \psi_{\lambda}\right] \mathcal{R}_{1}(\lambda) \psi_{\lambda}+\left[\mathcal{P}, \phi_{\lambda}\right] \mathcal{R}_{2}(\lambda) \phi_{\lambda}\right)^{-1}\right\| \leq C_{5}(\alpha) .
$$

Finally, since

$$
(\mathcal{P}-\lambda)^{-1}=\mathcal{R}^{\mathrm{app}}(\lambda) \circ\left(I+\left[\mathcal{P}, \psi_{\lambda}\right] \mathcal{R}_{1}(\lambda) \psi_{\lambda}+\left[\mathcal{P}, \phi_{\lambda}\right] \mathcal{R}_{2}(\lambda) \phi_{\lambda}\right)^{-1},
$$

we have that

$$
\left\|(\mathcal{P}-\lambda)^{-1}\right\| \leq\left\|\mathcal{R}^{\mathrm{app}}(\lambda)\right\|\left\|\left(I+\left[\mathcal{P}, \psi_{\lambda}\right] \mathcal{R}_{1}(\lambda) \psi_{\lambda}+\left[\mathcal{P}, \phi_{\lambda}\right] \mathcal{R}_{2}(\lambda) \phi_{\lambda}\right)^{-1}\right\| .
$$

Using (7.11) and (7.17), we find that (7.4) is true.

Proof of Proposition 7.1. The proof of (7.2) follows immediately from the GearhartPrüss Theorem and (7.4).

To prove (7.3) we note that since the resolvent of $\mathcal{P}$ is compact, the spectrum is discrete. Let $\lambda_{m} \in \mathbb{C}$ denote an eigenvalue with real part which equals $\mu_{m}$. There is clearly at least one eigenfunction associated with $\lambda_{m}$ which we denote by $u_{m}$. We prove (17.3) by observing that

$$
G(t) u_{m}=u_{m} e^{-\lambda_{m} t} .
$$

\section{Appendix A. Some Results Related to PSEUdo-SPeCtra}

We recall that, for any $\epsilon>0$, the $\epsilon$-pseudo-spectra of a linear operator $\mathcal{A}$ is defined by

$$
\Sigma_{\epsilon}(\mathcal{A})=\left\{\lambda \in \mathbb{C}:\left\|(\mathcal{A}-\lambda)^{-1}\right\|>\frac{1}{\epsilon}\right\},
$$

with the convention that

$$
\left\|(\mathcal{A}-\lambda)^{-1}\right\|=+\infty \quad \text { if } \lambda \in \sigma(\mathcal{A}) .
$$

We have

$$
\bigcap_{\epsilon>0} \Sigma_{\epsilon}(\mathcal{A})=\sigma(\mathcal{A})
$$

We define, for any accretive closed operator $\mathcal{A}$, for $\epsilon>0$, the $\epsilon$-pseudo-spectral abscissa

$$
\widehat{\alpha}_{\epsilon}(\mathcal{A})=\inf _{z \in \Sigma_{\epsilon}(\mathcal{A})} \operatorname{Re} z .
$$

We also define the growth bound of $\mathcal{A}$ by

$$
\widehat{\omega}_{0}(\mathcal{A})=\lim _{t \rightarrow+\infty} \frac{1}{t} \log \|\exp (-t \mathcal{A})\| .
$$

We mention also that

$$
\widehat{\alpha}_{\epsilon}(\mathcal{A}) \leq \inf _{z \in \sigma(\mathcal{A})} \operatorname{Re} z .
$$

Theorem A.1 (Gearhart-Prüss). Let $\mathcal{A}$ be a densely defined closed operator in a Hilbert space $X$ such that $-\mathcal{A}$ generates a contraction semi-group, and let $\widehat{\alpha}_{\epsilon}(\mathcal{A})$ and $\widehat{\omega}_{0}(\mathcal{A})$ denote the $\epsilon$-pseudo-spectral abscissa and the growth bound of $\mathcal{A}$, respectively. Then

$$
\lim _{\epsilon \rightarrow 0^{+}} \widehat{\alpha}_{\epsilon}(\mathcal{A})=-\widehat{\omega}_{0}(\mathcal{A}) .
$$

We refer to [11] for a proof. 


\section{ACKNOWLEDGEMENTS}

The first author was supported by NSF grant DMS 0604467. The work of the third author was partially supported by the National Natural Science Foundation of China grant no. 10871071 and no. 11171111, and the Science Foundations of the Ministry of Education of China no. 20110076110001.

\section{REFERENCES}

[1] M. Abramowitz and I. A. Stegun, Handbook of Mathematical Functions, Dover, 1972.

[2] J. Aguilar and J. M. Combes, A class of analytic perturbations for one-body Schrödinger Hamiltonians, Comm. Math. Phys., 22 (1971), 269-279. MR0345551 (49:10287)

[3] Y. Almog, The stability of the normal state of superconductors in the presence of electric currents, SIAM J. Math. Anal., 40 (2008), 824-850. MR2438788(2009i:35293)

[4] Y. Almog, B. Helffer, And X. B. Pan, Superconductivity near the normal state under the action of electric currents and induced magnetic fields in $\mathbb{R}^{2}$, Commun. Math. Phys., 300 (1) (2010), 147-184. MR2725185

[5] P. Bauman, H. Jadallah, and D. Phillips, Classical solutions to the time-dependent Ginzburg-Landau equations for a bounded superconducting body in a vacuum, J. Math. Phys., 46 (2005). MR2171207 (2006k:35268)

[6] S. J. Chapman, S. D. Howison, and J. R. Ockendon, Macroscopic models for superconductivity, SIAM Review, 34 (1992), 529-560. MR1193011(94b:82037)

[7] J. M. Combes and L. Thomas, Asymptotic behaviour of eigenfunctions for multiparticle Schrödinger operators, Comm. Math. Phys., 34 (1973), 251-270. MR0391792 (52:12611)

[8] E. B. Davies, Wild spectral behaviour of anharmonic oscillators, Bull. London Math. Soc., 32 (2000), 432-438. MR 1760807(2001e:47060)

[9] A. Dolgert, T. Blum, A. Dorsey, AND M. Fowler, Nucleation and growth of the superconducting phase in the presence of a current, Phys. Rev. B, 57 (1998), 5432-5443.

[10] Q. Du, M. D. Gunzburger, and J. S. Peterson, Analysis and approximation of the Ginzburg-Landau model of superconductivity, SIAM Review, 34 (1992), 54-81. MR:1156289 (93g:82109)

[11] K.-J. Engel And R. NAGel, One-parameter semigroups for linear evolution equations, Graduate Texts in Mathematics, vol. 194, Springer-Verlag, New York, 2000. MR1721989 (2000i:47075)

[12] S. Fournais and B. Helffer, Spectral Methods in Surface Superconductivity, Birkhäuser, 2009. MR2662319

[13] B. HelfFer, On pseudo-spectral problems related to a time dependent model in superconductivity with electric current, Confluentes Math., 3 (2) (2011), 237-251.

[14] B. I. IVlev AND N. B. Kopnin, Electric currents and resistive states in thin superconductors, Advances in Physics, 33 (1984), 47-114.

[15] T. Kato, Perturbation Theory for Linear Operators, Springer, Third Edition, 1980. MR0407617 (53:11389)

[16] M. Reed And B. Simon, Methods of Modern Mathematical Physics I, Functional Analysis, Academic Press Inc. (Harcourt Brace Jovanovich Publishers), New York, second edition, 1980. MR751959(85e:46002)

[17] J. Rubinstein And P. Sternberg, Formation and stability of phase slip centers in nonuniform wires with currents, Physica C-Superconductivity and Its Applications, 468 (2008), 260-263.

[18] J. Rubinstein, P. Sternberg, And Q. MA, Bifurcation diagram and pattern formation of phase slip centers in superconducting wires driven with electric currents, Phys. Rev. Lett., 99 (2007).

[19] J. Rubinstein, P. Sternberg, and K. Zumbrun, The resistive state in a superconducting wire: bifurcation from the normal state, Arch. Rational Mech. Anal., 195 (2010), 117-158. MR:2564470 (2010m:82112)

[20] B. Simon, Schrödinger semigroups, Bull. Amer. Math. Soc. (N.S.), 7 (1982), 447-526. MR670130 (86b:81001a) 
[21] A. G. Sivakov, A. M. Glukhov, A. N. Omelyanchouk, Y. Koval, P. Müller, and A. V. Ustinov, Josephson behavior of phase-slip lines in wide superconducting strips, Phys. Rev. Lett., 91 (2003), art. no. 267001.

[22] M. Tinkham, Introduction to Superconductivity, McGraw-Hill, 1996.

[23] D. Y. Vodolazov, F. M. Peeters, L. Piraux, S. Matefi-Tempfli, and S. Michotte, Current-voltage characteristics of quasi-one-dimensional superconductors: An s-shaped curve in the constant voltage regime, Phys. Rev. Lett., 91 (2003).

Department of Mathematics, Louisiana State University, Baton Rouge, Louisiana 70803

E-mail address: almog@math.1su.edu

Laboratoire de Mathématiques, Université Paris-Sud 11 et CNRS, BÂt 425, 91405 Orsay Cedex, France

E-mail address: Bernard.Helffer@math.u-psud.fr

Department of Mathematics and Center for PDE, East China Normal University, Shanghai 200062, People's Republic of China

E-mail address: xbpan@math.ecnu.edu.cn 